\title{
On the Construction of Raindrop Erosion Maps for Steel
}

\author{
J. A. Sloan, M. M. Stack \\ Department of Mechanical and Aerospace Engineering, \\ University of Strathclyde, Glasgow UK
}

\section{Acronyms}

AFP

ATL

CFRP

EDS

EWEA

GFRP

GW

$\mathrm{mm}$

$\mathrm{ml}$

$\mathrm{mg}$

NDI

RETR

SEM

UDRI

UK

WARER
Automated fibre placement

Automated tape layup

Carbon fibre reinforcement plastic

Energy dispersive spectroscopy

European wind energy association

Glass fibre reinforcement plastic

Giga Watts

Millimeters

Milliliters

Milligrams

Non-destructive inspection

Rain erosion test rig

Scanning electron microscopy

University of Dayton Research Institute

United Kingdom

Whirling arm rain erosion rig

\section{Nomenclature}

$C_{1} \quad$ Speed of sound in liquid $\left(\mathrm{ms}^{-1}\right)$

$\mathrm{C}_{\mathrm{s}} \quad$ Speed of sound in solid $\left(\mathrm{ms}^{-1}\right)$

$C_{\rho} \quad$ Speed of sound in undistributed density $\left(\mathrm{ms}^{-1}\right)$

d Rain droplet diameter $(\mathrm{mm})$

F Impact force (N)

$\mathrm{m} \quad$ Mass of raindrop $(\mathrm{kg})$

pl $\quad$ Density of liquid $\left(\mathrm{kg} / \mathrm{m}^{3}\right)$

po $\quad$ Undistributed density of the fluid $\left(\mathrm{kg} / \mathrm{m}^{3}\right)$

$\rho_{\mathrm{s}} \quad$ Density of solid $\left(\mathrm{kg} / \mathrm{m}^{3}\right)$

$\mathrm{V} \quad$ Impact velocity $\left(\mathrm{ms}^{-1}\right)$

$V_{0} \quad$ Impact velocity $\left(\mathrm{ms}^{-1}\right)$

\section{Abstract}

Raindrop erosion is a significant materials limiting issue. It can affect materials for transportation and renewable energy converters such as wind turbines as well as all external structures used in the construction industries. In such cases, the raindrop effects can occur over a wide range of impact velocities and particles sizes.

In understanding tribological variable effects, it is useful to study using reference materials. This is because the reference material is well characterized, from previous knowledge of the tribological and corrosion patterns. Further, the well established chemical composition and stochiometry of the corrosion products provide some background understanding of how effectively, during tribological action, the surface scale adheres to the substrate.

In this study, a carbon steel was used as a reference to study the effects of velocity and drop diameter in a whirling arm erosion test rig. The results were used to establish the conditions where corrosion was accelerated and the environments where aerodynamic effects may have resulted in 
droplets being deflected the surfaces. Erosion maps were generated to illustrate such mechanisms based on the results.

\section{Introduction}

Society today aims towards a future where renewable energy resources dominate over productions from fossil fuels and nuclear fission power resources. With weather patterns changing across the globe, speculations of global warming being the major contributor has led the UK government to make drastic legislations to meet a cleaner means of power production. Several Energy White Papers, for example that published in 2003, concluded that if the UK is to achieve a reduction in carbon emissions of the required scale then renewable energy will need to contribute at least 30$40 \%$ of our electricity generation.'

The UK is one of the optimum locations in the world for wind power. By way of illustration of the vast rate of growth in renewable energies, the UK electricity generated by renewable energy increased to over 30\% 2019 alone."i To accomplish these targets in renewable energies, large investments have been made to improve the efficiencies and overall power contributions from all renewable energy resources. These targets have thus led to an increase in wind turbine numbers and technological advance in the wind energy sector. ${ }^{\text {.ii }}$

Developing wind turbines and improving the efficiency is a very complex procedure with many environmental and tribological factors to take into consideration. Extreme winds and gales cause bending and cyclic loading on the frame and blades of the wind turbine. Abundant rain and hailstone showers affect the aerodynamic efficiency of the blades due to leading edge erosion. As every continent in the world has different environmental conditions, factors such as extreme temperatures, snow/icing and ultra violet light exposure must be brought into consideration when developing these turbines. Off-shore wind farms may have more issues in the long term due to high maintenance costs and the generally large blade lengths in such conditions. This brings a disadvantage of greater wind tip velocities on the blade that accelerates erosion of the leading edge and rapidly decreases the aerodynamic efficiency.

Historically there has been less attention given to the effects of liquid impacts and Field (1999) states it was not until steam turbine blades were found to erode that sparked interest in this subject. ${ }^{\text {iv }}$ Research in this field became more popular over the decades, as aircraft was getting faster more research was focused on the erosion of aircraft wings. Much research has been focused on the dynamics behind the rain drop impact explaining how damage on the blades occur.

Liquid impacts consist of two main stages, firstly the liquid behaves in a compressible manner generating the so called water hammer pressure. Water hammer pressure is a pressure surge or wave created when a fluid collides with a surface causing it to stop abruptly. These high pressures cause the greatest damage on the surface due to liquid impacts and are maintained while the edge of the contact area between the impacting liquid and the solid moves supersonically with respect to the shock speed. ${ }^{v}$ The interaction of these waves are very complex, depending upon the impact conditions and material properties of the impact surface. A Rayleigh wave is created on impact and 
travels along the solid surface. The compressed liquid wave in the droplet after a short time duration after impact, extends towards and past the contact periphery between the droplet and the surface. After this point lateral jetting or more commonly known splashing of the droplet occurs. ${ }^{5}$

The water hammer pressure mentioned previously can be calculated using the equations below; ${ }^{4}$

$P=\rho_{o} c_{o} V_{o}-$ Equation 1

$P=\frac{V \rho_{l} c_{l} \rho_{s} c_{s}}{\rho_{l} c_{l}+\rho_{s} c_{s}}-$ Equation 2

(Equation 1) is a simple representation of the water hammer pressure and was originally used to calculate water hammer pressures present in piping systems, based on quite straight forward assumptions. Dear and Field proposed a modified water hammer equation which takes into consideration not only the pressure through the liquid but also the colliding surface. ${ }^{5}$ The subscripts in (Equation 2) (I) and (s) refer to liquid and solid bodies respectively. An instantaneous approximation of the impact force created by the rain droplet on the surface can be calculated from (Equation 3) stated below;

$F=\frac{m V^{2}}{d}-$ Equation 3

A single rain drop has very little effect on a solid surface; it is the repetitive impacts from rainfall over long periods that cause permanent damage. When the rain drop makes initial contact with the surface, the water hammer pressure produced causes small cavities to gradually form. These cavities were proven to be caused by the formation of pressure waves. Further impacts deepen these cavities before giving rise to stress concentrations in the material that cause more of the material to be removed. The erosion process causes work hardening and further crack growth which leads to greater loss of surface material. ${ }^{\text {vi } A}$ more recent researcher Hattori (2010) proposed that the fracture toughness and fatigue crack removal was the main mechanism involved in the erosion process. ${ }^{\text {vii }}$

Damage on wind turbine blades is known as leading edge erosion in the wind industry and can be explained as pitting and gouging around the edge of the blade. Damage of the composite on the leading edge can reduce the aerodynamic efficiency after only two years in service. ${ }^{\text {vii }}$ In an attempt to address this issue of leading edge erosion, companies are researching and developing new materials for protecting the leading edge. Applying elastomeric materials such as tapes to the blades are becoming very effective methods of improving the blades resistance to erosion.

In heavy rainfall impact velocities reach up to $100 \mathrm{~ms}^{-1}$, previous work quotes a single rain drop can impact pressures of $140 \mathrm{MPa}$ on a surface, with repetitive bombardment these impacts can cause a lot of damage. A $20 \%$ reduction in annual energy output due to this phenomenon has been estimated. ${ }^{\text {ix }}$ This highlights the engineering challenge behind reducing this leading edge erosion. In relation to the leading edge erosion, the free fall velocity of a raindrop will have an impact on the overall collision velocity. Keegan et al. shows the free fall velocity only has a minor role in the magnitude of the impact velocity when compared to blade tip velocities. It is possible, through 
vector calculations to establish an approximation for the impact velocity for a given rain and turbine conditions, through a whole rotor sweep. The maximum impact velocity occurs at $270^{\circ}$ on the rotor sweep and is the resultant of the free fall velocity and the tip speed of the blade.

Raindrop diameters vary with respect to climate conditions under which they are formed and conditions of transport in the air. Typical raindrop diameters are commonly in the range between $0.5 \mathrm{~mm}$ and $5 \mathrm{~mm}$, above this maximum diameter droplets are very unstable and begin to fragment. Kubilay et al. produced a graph for the probability density for the raindrop diameters using the equations derived by Best, ${ }^{x, x i}$ as shown in Figure 1.

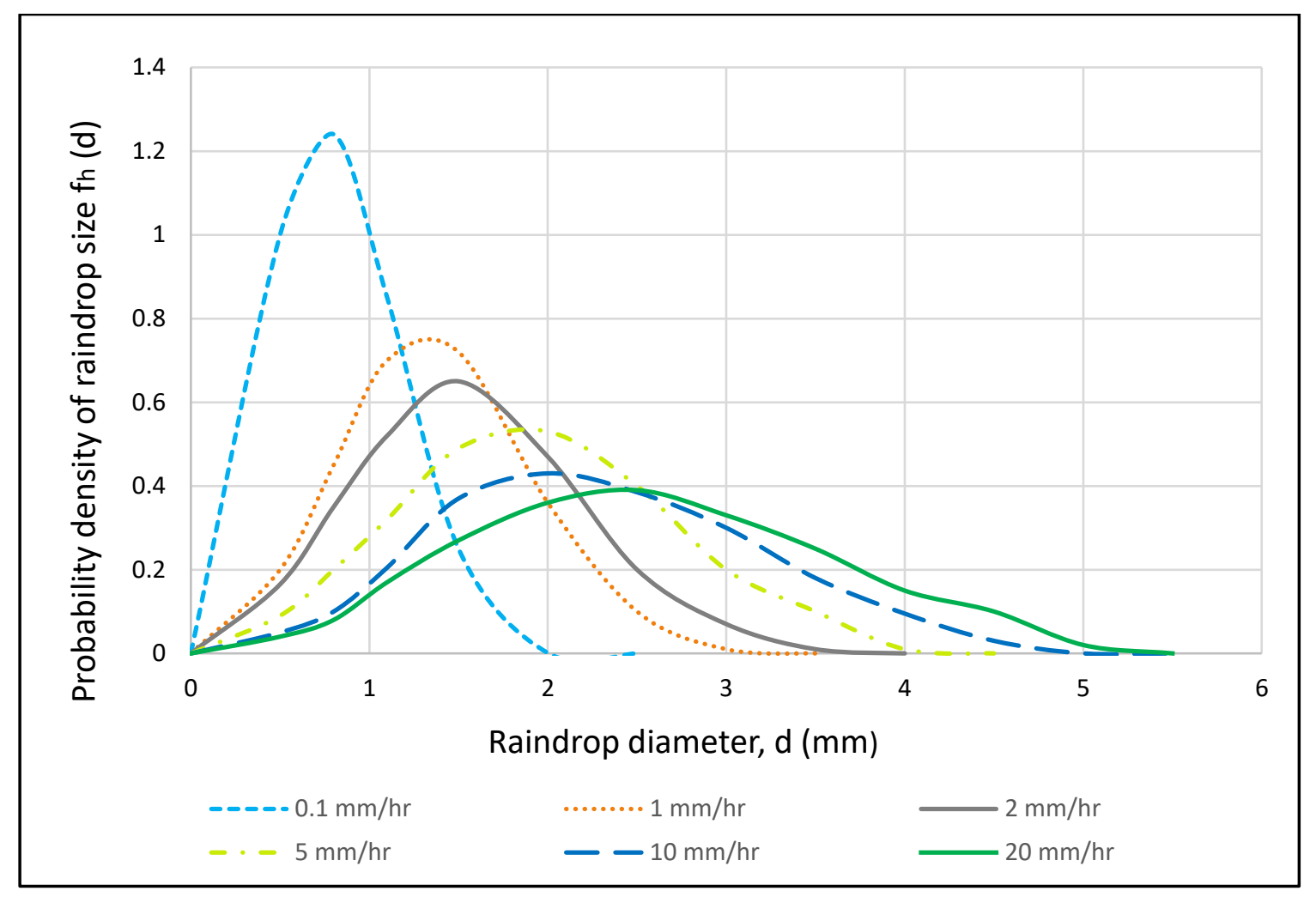

Figure 1: Probability density of raindrop size. Calculated using equation from ${ }^{\text {xii }}$

As can be observed in the probability graph above, Fig. 1, the average rain droplet diameters during moderately calm rain fall range between $0.5 \mathrm{~mm}$ and $3 \mathrm{~mm}$. The peak or most common raindrop diameters in all variations of rain conditions tested and recorded in the graph, range between $1.4 \mathrm{~mm}$ and $3 \mathrm{~mm}$ respectively, ignoring the $0.1 \mathrm{~mm} / \mathrm{h}$ rainfall.

In the present study, the erosion is evaluated over a range of velocities and particle sizes. Morphological study of the damage surfaces is carried out. Based on such observations and mass loss data, erosion maps are constructed based on the results. 


\section{Experimental Details}

\subsection{Initial rain erosion test rig apparatus}

The original apparatus for the rain erosion test rig (RETR) was developed as a master's project and has slowly been altered and calibrated through other projects. The basic concept of the rig uses horizontal arms on which test specimens are attached and made to rotate in an enclosed chamber under wind turbine simulated conditions (Figure 2). The velocity and rain droplet diameters are varied to simulate different rain conditions.

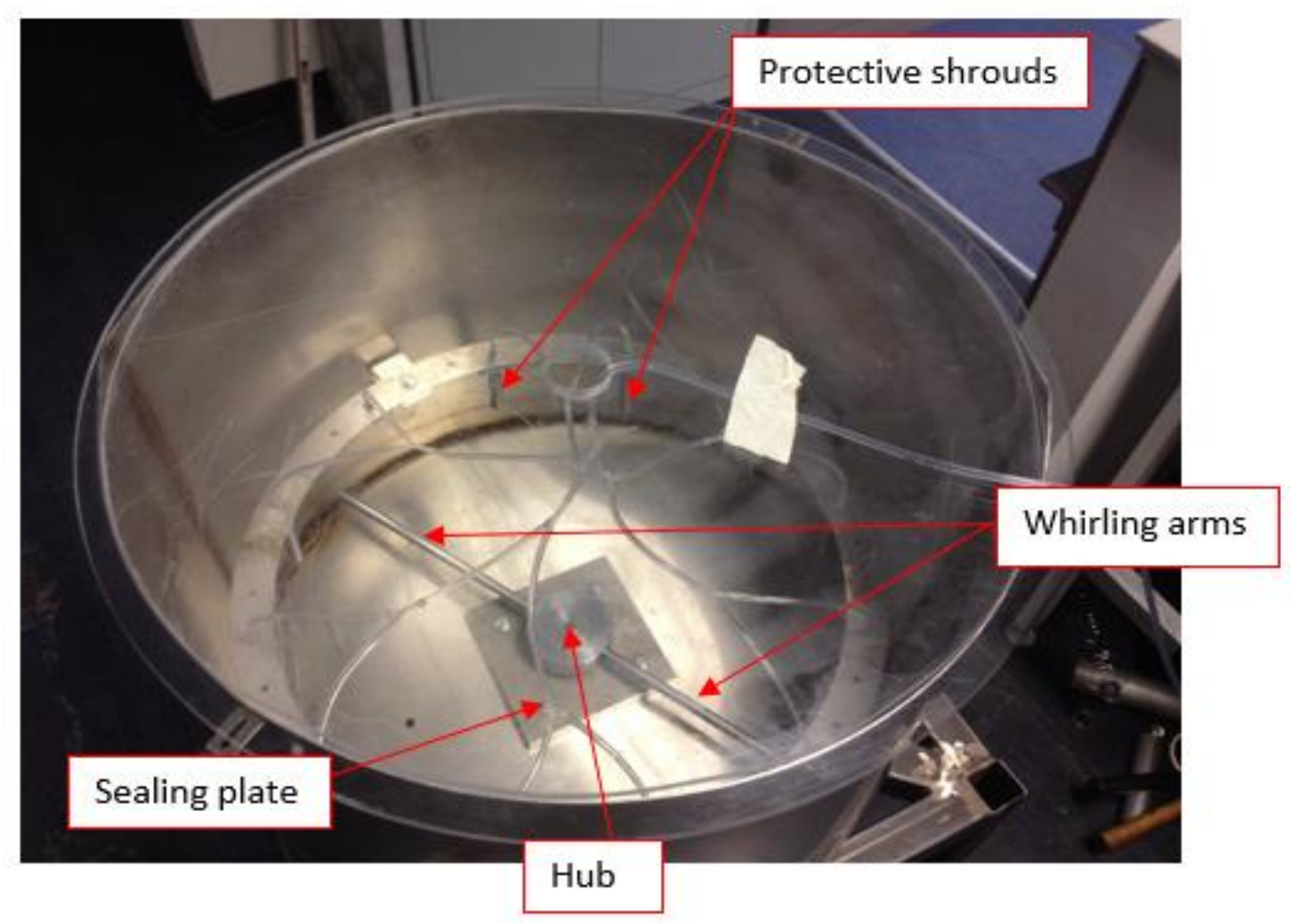

Figure 2: Rain erosion test rig after developments

Powered by an AC motor, a pulley and belt system provides the rotational motion to the hub on which the whirling arms and mounts are connected. The rotational velocity is controlled safely by an $A C$ invertor. A relationship between the rotational velocity of the motor drive shaft and the output frequency of the inverter provides an easy method of controlling the rotational velocity.

Developments were undertaken to increase the rotational velocity of the (RETR). During developments however severe corrosion damage on the bearings resulted in the drive shaft to twist. The damaged caused a distinct wobble on the hub redeeming it too unstable for increasing the rotational velocity before repairs were completed. 
A $200 \mathrm{CM}$ series peristaltic pump, with a flow rate ranging between $10 \mathrm{~mL} / \mathrm{min}$ and $40 \mathrm{~mL} / \mathrm{min}$ was used to control the flow of water to the needles. This type of pump is an accurate method for controlling the flow rate, depending on the test conditions. A flow rate equivalent to $25.4 \mathrm{mmh}^{-1}$ of rain is widely used by researchers as the worst case scenario of extremely heavy rain fall common in tropical regions.

The needle gauge evaluation was as follows:

$V_{S}=\frac{4}{3} \pi r^{3}-$ Equation 4

$A=\pi\left(r_{o}\right)^{2}-\pi\left(r_{i}\right)^{2}-$ Equation 5

$V_{r}=A \times 0.0254-$ Equation 6

$N_{r}=\frac{V_{r}}{V_{s}}-$ Equation 7

The volume of water required for the test was calculated assuming the average raindrop is $2 \mathrm{~mm}$ in diameter and spherical in shape. (Equation 4) gives a volume of the raindrop to be $4.19 \times 10^{-9} \mathrm{~m}^{3}$. The test specimens during these tests had the same diameter of $30 \mathrm{~mm}$ used in previous work. The hub and whirling arms produce a rotating radius of $0.3015 \mathrm{~m}$. The revolving path of the test specimens will then have an outer rotating radius of $0.3155 \mathrm{~m}$ and an inner radius of $0.2855 \mathrm{~m}$ which gives an annular area of $0.0566 \mathrm{~m}^{2}$ according to (Equation 5).

As the desired rain fall rate for the tests are to be $25.4 \mathrm{mmh}^{-1}$, the required rainfall volume for one hour is $1.44 \times 10^{-3} \mathrm{~m}^{3} \mathrm{~h}^{-1}$ and was calculated using (Equation 6). With an average rain drop volume of $4.19 \times 10^{-9} \mathrm{~m}^{3}$ and the rain fall volume over one hour $1.44 \times 10^{-3} \mathrm{~m}^{3}$ from (Equation 7), 343,675 raindrops will be produced per hour and 95 raindrops per second were required.

Referring to section (1.5) the most abundant raindrop diameters for all rainfall conditions ranged between $1.4 \mathrm{~mm}$ and $3.0 \mathrm{~mm}$. For this reason testing in this project focused on raindrop diameters between $1.8 \mathrm{~mm}$ and $3.0 \mathrm{~mm}$, shown in (Table 1), which displays the various needle gauges used and the corresponding raindrop diameters they produce.

Table 1: Variation of raindrop diameter with needle gauge

\begin{tabular}{|l|l|l|}
\hline Needle gauge & Needle colour & Raindrop size $(\mathbf{m m})$ \\
\hline 19 & White & 3.0 \\
\hline
\end{tabular}




\begin{tabular}{|l|l|l|}
\hline $\mathbf{2 1}$ & Green & 2.8 \\
\hline $\mathbf{2 3}$ & Blue & 2.5 \\
\hline $\mathbf{2 5}$ & Orange & 2.0 \\
\hline $\mathbf{3 0}$ & Yellow & 1.8 \\
\hline
\end{tabular}

\subsection{Test Specimen Holder}

Initially a wear resistant adhesive was used to attach the test specimen onto the mount. At the end of the test a new specimen had to replace the existing one so a comparison could be made under different test conditions. Many methods were attempted to remove the specimen from the mount, examples being heat treatment and the development of a jig, which was an attempt at removing the test specimen by force. Both methods were unsuccessful and kept destroying the mounts and damaging the test sample. For this reason previous testing resulted in a new mount being used for each test.

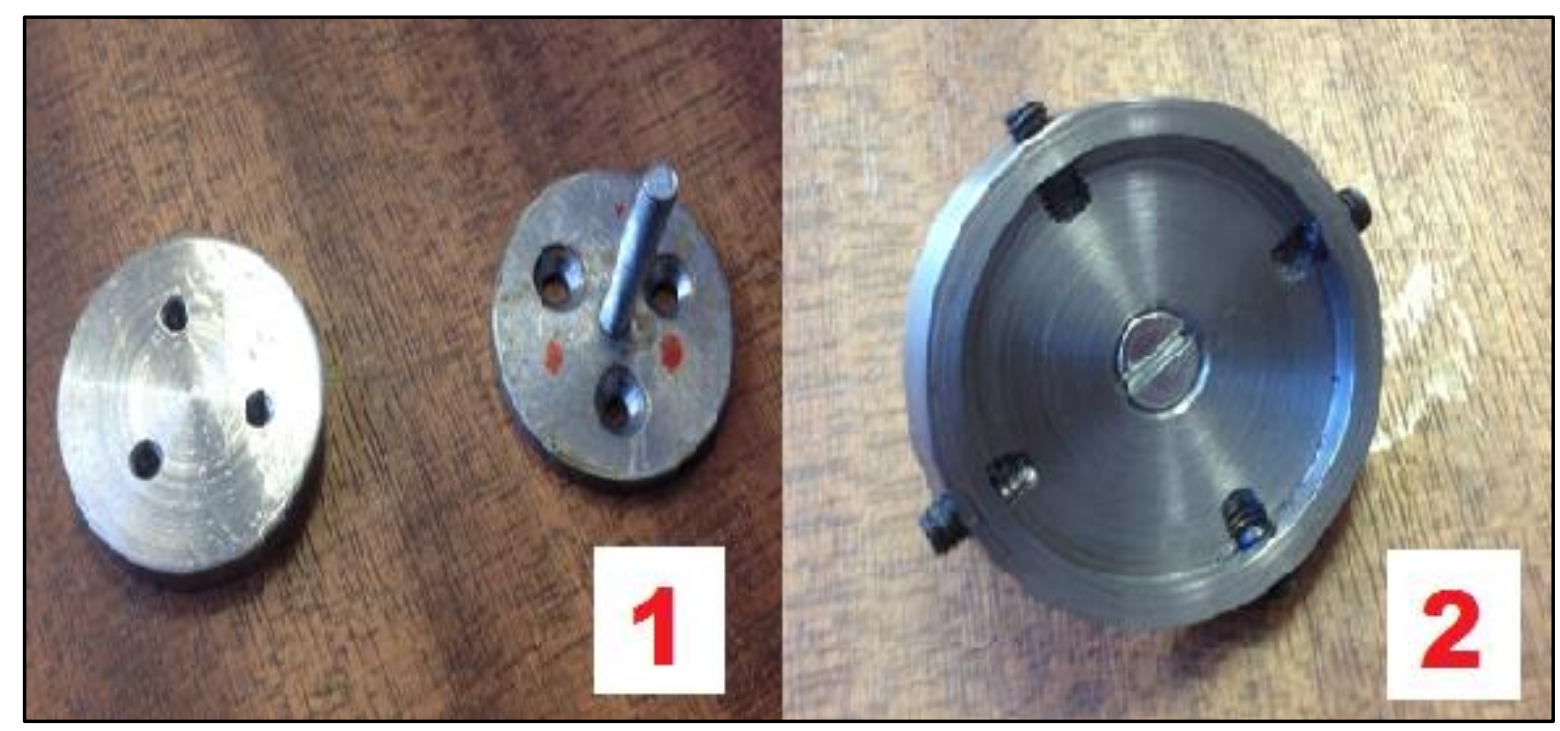

Figure 3: a) Test specimen holder design No. $1 \quad$ b) Test specimen holder design No. 2

New designs of the test specimen holder were created and tested. The new design was developed to run tests quicker and more efficiently. By being able to remove the samples at the end of testing allowed a comparison of the corrosion and wear scars for various different test conditions. Figure 3 shows the two final designs that were sent to the workshop for construction. Design number 1 demonstrated the most potential with a strong connection due to three small screws. Designs had to be made to produce minimal wear on the test specimens as any mass loss would affect the final results of the tests; for this reason design 1 was used as the mount during this project. 


\subsection{Initial testing}

$V=\omega \times r \times \frac{2 \pi}{60}-$ Equation 8

Initial tests were carried out on the rig before starting preliminary testing using raindrop impacts, as some major developments were undertaken. The tests were used to confirm the rotational velocity of the rig was the same as before developments. The rotational velocity of the whirling arms for a given frequency from the $A C$ invertor to the motor was measured using an optical tachometer, results are displayed in (Table 2). The linear velocity of the test specimen was calculated using (Equation 8). The velocities of the rig are seen to be slightly lower than before developments. Reason being the sealing plate rubs against the drive shaft causing a small frictional torque.

Table 2: Velocities measured before and after development

\begin{tabular}{|l|l|l|l|}
\hline $\begin{array}{l}\text { Frequency } \\
(\mathbf{H z})\end{array}$ & $\begin{array}{l}\text { Average rotational } \\
\text { velocity }\end{array}$ & $\begin{array}{l}\text { Linear Velocity } \\
(\mathrm{rad} / \mathrm{s})\end{array}$ & $\begin{array}{l}\text { Linear velocity of initial } \\
\text { rig design }(\mathrm{m} / \mathrm{s})\end{array}$ \\
\hline $\mathbf{1 0}$ & 517.6 & 16.3 & 17 \\
\hline $\mathbf{2 0}$ & 1044 & 32.9 & 35 \\
\hline $\mathbf{3 0}$ & 1516 & 47.7 & 51 \\
\hline $\mathbf{4 0}$ & 1922 & 60.5 & 66 \\
\hline $\mathbf{5 0}$ & 2197 & 69.1 & 78 \\
\hline
\end{tabular}

\subsection{SEM and EDS Analysis}

Specimens were analysed before and after testing by SEM allowing high resolution images of their topography. Analysis of the images will show whether erosion has taken place. An elemental analysis using EDS was also carried out on the mild steel specimens to indicate the elemental compositions on the surface of the specimen before and after testing. The results display a percentage of each element, beneficial in determining any oxides or indications of a corrosive process.

\subsection{Impact Testing}

\subsubsection{Mild Steel Specimens}

Mild steel is being used as a reference material to calibrate the rig to working conditions that can erode GFRP and CFRP composite materials. Currently the rig cannot produce enough impact energy 
to erode the surface of fibre reinforced-plastics. The rig was modified to run at faster velocities to produce larger impact energies that will erode the surface of the composites. Initially test specimens were made from $2 \mathrm{~mm}$ thick mild steel with a circular diameter of $30 \mathrm{~mm}$. In-light of the new mount the test specimens were altered to have a thickness of $5 \mathrm{~mm}$, with three $\mathrm{M} 3$ holes drilled at 120 degree intervals, $3 \mathrm{~mm}$ deep, on the bottom surface of the test specimen. An image of the test samples and mount can be observed in Figure 3[a].

\section{Results}

\subsection{Preliminary Testing of Mild Steel}

Preliminary testing was carried out to verify the new developments had not affected the rig as a rain erosion test rig. Testing was carried out using gauge 23 needles to produce a $2.5 \mathrm{~mm}$ diameter raindrop at a wind velocity of $32.9 \mathrm{~ms}^{-1}$. Both the mount and test specimen were weighed together before and after the test. The initial testing phase showed a large variation in mass loss and also mass gain in the range of $15 \mathrm{mg}$ per 20 minute interval. Comparison to previous work on liquid impact testing using mild steel the results were showing excessive mass loss and gains. ${ }^{14,17}$

1. Between the surfaces of the test specimen and mount was a large build-up of corrosive matter, perhaps caused by the velocity of the whirling arms pulling water and mass particles along the surface of the test specimen and into the gap cavity. There is also a smaller effect from galvanic coupling between the two metals which could be detrimental to the increase in corrosion.

2. The updraft caused from the increasing velocity of the whirling arms affected the trajectory of the rain droplets. Water droplets were seen collecting at the side of the rig walls at faster velocities whereas at lower velocities this was not observed. Signifying many droplets may not be coming into contact with the test material.

Corrosion between the surfaces denotes other reactions are occurring rather than solely on the top surface of the sample being examined. To improve the accuracy of the results this variable had to be removed, accomplished by the use of a non-corrosive coating. A thin layer of varnish covered the mounts and the underside of the test specimens where these unwanted reactions were taking place.

With implantation of the varnish on the mount and underside of the test specimen, further observations were made.

1. There was still a large unexplained increase in mass

2. Varnish successfully removed any sign of corrosion from in between the test specimen.

Having separated the test specimen from the mount small water droplets were discovered on the varnish in-between the surfaces explaining the unusual variations in mass loss and gain. Another important variable discovered when removing the test specimen was after it had been handled, many finger prints on the surface varied the mass by $0.25 \mathrm{mg}$ to $0.5 \mathrm{mg}$. 
Further tests were carried out wearing gloves at all times when handling the test specimens. The test specimens were cleaned with acetone at the beginning of each test to remove dust particles from the lab or oil residue from machining. For each weighing the specimen was dismantled from the mount and dried to remove the water on the inner surface. The test subject was then weighed 3 times and an average mass was taken to get the most accurate result possible, as the scales were not in pristine condition. Once most of the variables were eliminated, tests started displaying consistent mass losses.

\subsection{Erosion of mild steel with varying linear velocity}

Tests were undertaken to study the effect varying the shaft linear velocity has on the mass loss of mild steel. The results show a gradual mass loss as the linear velocity increases, tabulated in Table 4 and discussed in section 4.2 .

Table 4: Mass loss due to varying linear velocity

\begin{tabular}{|l|l|l|l|l|}
\hline $\begin{array}{l}\text { Test duration } \\
\text { (minutes) }\end{array}$ & $\begin{array}{l}\text { Invertor } \\
\text { setting }\end{array}$ & $\begin{array}{l}\text { Shaft linear } \\
\text { velocity } \mathbf{( m / s )}\end{array}$ & $\begin{array}{l}\text { Total mass loss of } \\
\text { subject } \mathbf{1}(\mathbf{m g})\end{array}$ & $\begin{array}{l}\text { Total mass loss of } \\
\text { subject 2 (mg) }\end{array}$ \\
\hline $\mathbf{0}$ & 0 & 0 & 0 & 0 \\
\hline $\mathbf{2 0}$ & 10 & 16.3 & 0.5 & 0.3 \\
\hline $\mathbf{4 0}$ & 20 & 32.9 & 0.6 & 0.4 \\
\hline $\mathbf{6 0}$ & 30 & 47.7 & 1 & 0.7 \\
\hline $\mathbf{8 0}$ & 40 & 60.5 & 1.1 & 0.9 \\
\hline
\end{tabular}

\subsection{Erosion of steel specimens with changing diameter}

The mass loss was taken using a set of weighing scales able to measure to an accuracy of $0.1 \mathrm{mg}$. Results for varying rain droplet diameters at the required velocities are displayed in Tables 5, 6, 7 and 8 respectively. Due to jets of water forming from the end of the needles and producing an inconstancy of raindrops; impact testing on rain drop diameters $1.8 \mathrm{~mm}$ and $3.0 \mathrm{~mm}$ could not be undertaken, as specified in section 5.4 .

Results show tests at $16.3 \mathrm{~ms}^{-1}$ encountered the largest mass loss. According to previous work these results are very peculiar. ${ }^{14,17}$ Graphs in Figures $11,12,13$ and 14 in (section 4.3) respectively explain the results of varying rain drop diameter in more detail. Observations from the tables show a reduction in the overall mass loss over the 60 minutes of testing at higher velocities compared to impact velocity of $16.3 \mathrm{~ms}^{-1}$. 
Table 5: Mass loss of steel samples at $16.3 \mathrm{~ms}^{-1}$ with changing raindrop diameter

\begin{tabular}{|l|l|l|l|}
\hline & \multicolumn{3}{|l|}{ Mass loss of steel at specified rain drop diameters (mg) } \\
\hline Time (min) & $\mathbf{2 m m}$ & $\mathbf{2 . 5 m m}$ & $\mathbf{2 . 8 m m}$ \\
\hline $\mathbf{0}$ & 0 & 0 & 0 \\
\hline $\mathbf{2 0}$ & 0.4 & 0.4 & 0.5 \\
\hline $\mathbf{4 0}$ & 0.9 & 0.7 & 0.2 \\
\hline 60 & 1.5 & 1.2 & 0.7 \\
\hline
\end{tabular}

Table 6: Mass loss of steel samples at $32.9 \mathrm{~ms}^{-1}$ with changing raindrop diameter

\begin{tabular}{|l|l|l|l|}
\hline \multicolumn{2}{|l|}{ Mass loss of steel at specified rain drop diameters (mg) } \\
\hline Time (min) & $\mathbf{2 m m}$ & $\mathbf{2 . 5 m m}$ & $\mathbf{2 . 8 m m}$ \\
\hline $\mathbf{0}$ & 0 & 0 & 0 \\
\hline $\mathbf{2 0}$ & 0.6 & 0.2 & 0.2 \\
\hline $\mathbf{4 0}$ & 0.9 & 0.3 & 0 \\
\hline 60 & 0.9 & 0.9 & 0.4 \\
\hline
\end{tabular}

Table 7: Mass loss of steel samples at $47.7 \mathrm{~ms}^{-1}$ with changing raindrop diameter

\begin{tabular}{|l|l|l|l|}
\hline & \multicolumn{3}{|l|}{ Mass loss of steel at specified rain drop diameters $(\mathbf{m g})$} \\
\hline Time (min) & $\mathbf{2 m m}$ & $\mathbf{2 . 5 m m}$ & $\mathbf{2 . 8 m m}$ \\
\hline $\mathbf{0}$ & 0 & 0 & 0 \\
\hline $\mathbf{2 0}$ & -0.1 & 0.8 & 0.3 \\
\hline $\mathbf{4 0}$ & 0.4 & 1.0 & 0.8 \\
\hline
\end{tabular}




\begin{tabular}{|l|l|l|l}
\hline 60 & 0.5 & 1.1 & 0.9
\end{tabular}

Table 8: Mass loss of steel samples at $60.5 \mathrm{~ms}^{-1}$ with changing raindrop diameter

\begin{tabular}{|l|l|l|l|}
\hline & \multicolumn{3}{|l|}{ Mass loss of steel at specified rain drop diameters (mg) } \\
\hline Time (min) & $\mathbf{2 m m}$ & $\mathbf{2 . 5 m m}$ & $\mathbf{2 . 8 m m}$ \\
\hline $\mathbf{0}$ & 0 & 0 & 0 \\
\hline $\mathbf{2 0}$ & 0.2 & 0.3 & 0.2 \\
\hline $\mathbf{4 0}$ & 0.4 & 0.2 & 0.5 \\
\hline 60 & 0.5 & 0.6 & 1.0 \\
\hline
\end{tabular}

As the velocity is further increased to $47.7 \mathrm{~ms}^{-1}$ there is still no signs of an increasing mass loss rate (Table 8). A transition can be spotted in the results where the larger raindrop diameters produce a greater mass loss at higher velocities in comparison to the lower velocities (Table 8).

\subsection{SEM images and EDS results}

A S3700 (Hitachi, Japan) Tungsten Filament Scanning Electron Microscope (SEM) was used to examine the surface of the mild steel. Initial SEM micrographs prior to impact testing display a flat surface as seen in Figure 4. The black spots seen on the surface of the specimens were found to be impurities of manganese sulphide using energy dispersive spectroscopy (EDS). High levels of oxygen, manganese and sulphur are common in steels, as when the steel is in molten form it is very soluble to oxygen which defuses into the slag (a residue on the surface of the liquid steel) forming the manganese sulphide. These inclusions were considered to have negligible effect on the tests being run in this project but in many cases these inclusion affect the malleability of the steel. Since all test specimens were machined from the same piece of mild steel they were all very similar in comparison to Figure 4. 


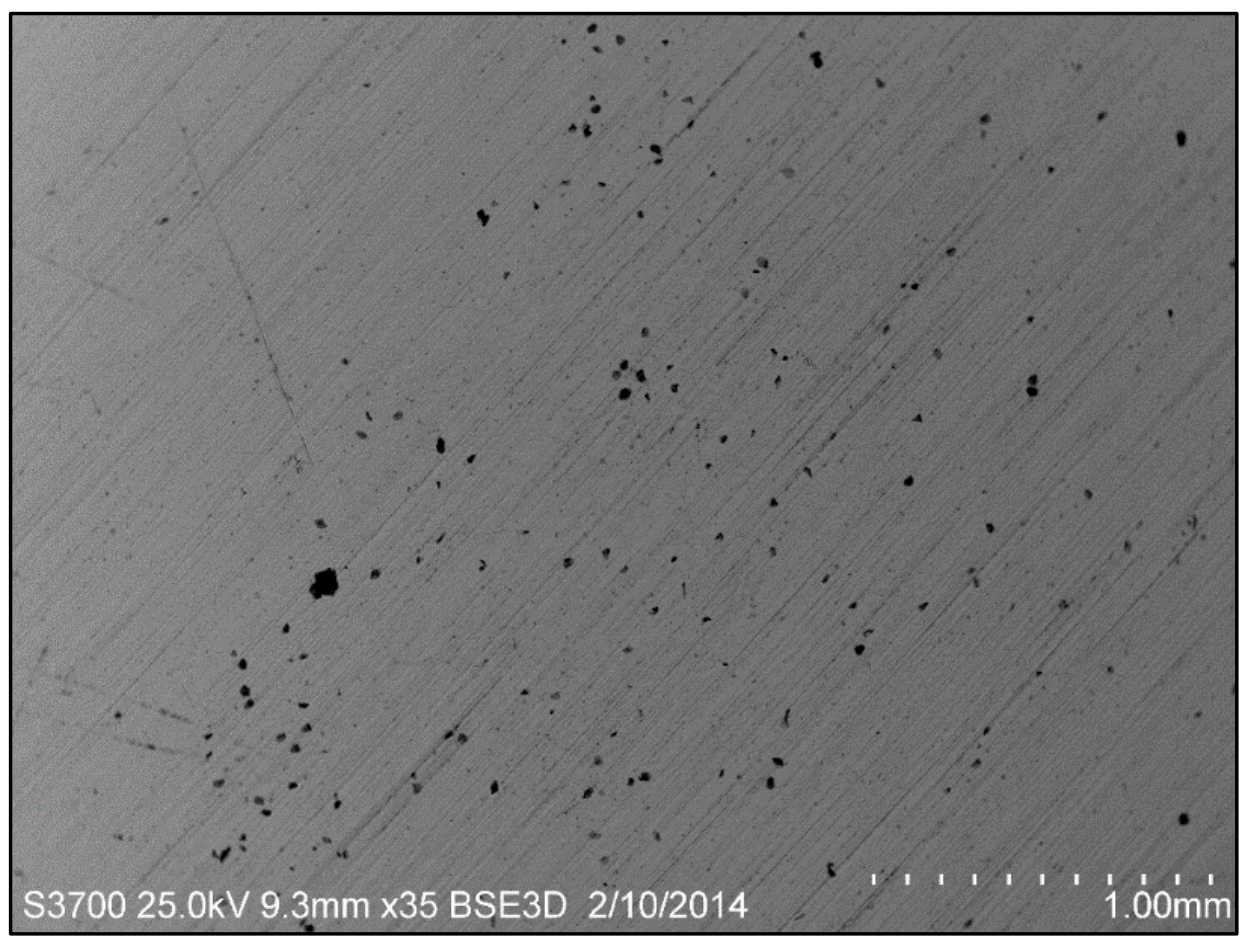

Figure 4: Test specimen prior to impact testing

(Table 9) was used to emphasise the composition of elements in the mild steel prior to impact testing. Carbon is ignored from the EDS results as the surface of the specimen is coated with a thin layer of carbon prior to scanning, consequentially the grade of steel is unknown.

Table 9: Elements of the mild steel surface prior to impact testing

\begin{tabular}{|l|l|}
\hline Element & Weight\% \\
\hline Iron, Fe & 99.3 \\
\hline Manganese, Mn & 0.7 \\
\hline
\end{tabular}

The micrograph in Figure 5 was taken from a small section of the same sample after 80 minutes of liquid impact testing. Comparison of the two images indicate a change on the surface of the steel due to rain drop impacts. EDS was used to determine the chemical compositions of the testing surface of the mild steel. The results in Table 10 show high oxygen levels, indicating the presence of an iron oxide layer. Elements such as chlorine and sodium may be explained as impurities on the surface of the steel from the tap water used during testing. Nickel should not be seen on the surface of the mild steel and $10 \%$ is an extremely high approximation and was narrowed down to an inaccuracy with the EDS result. 


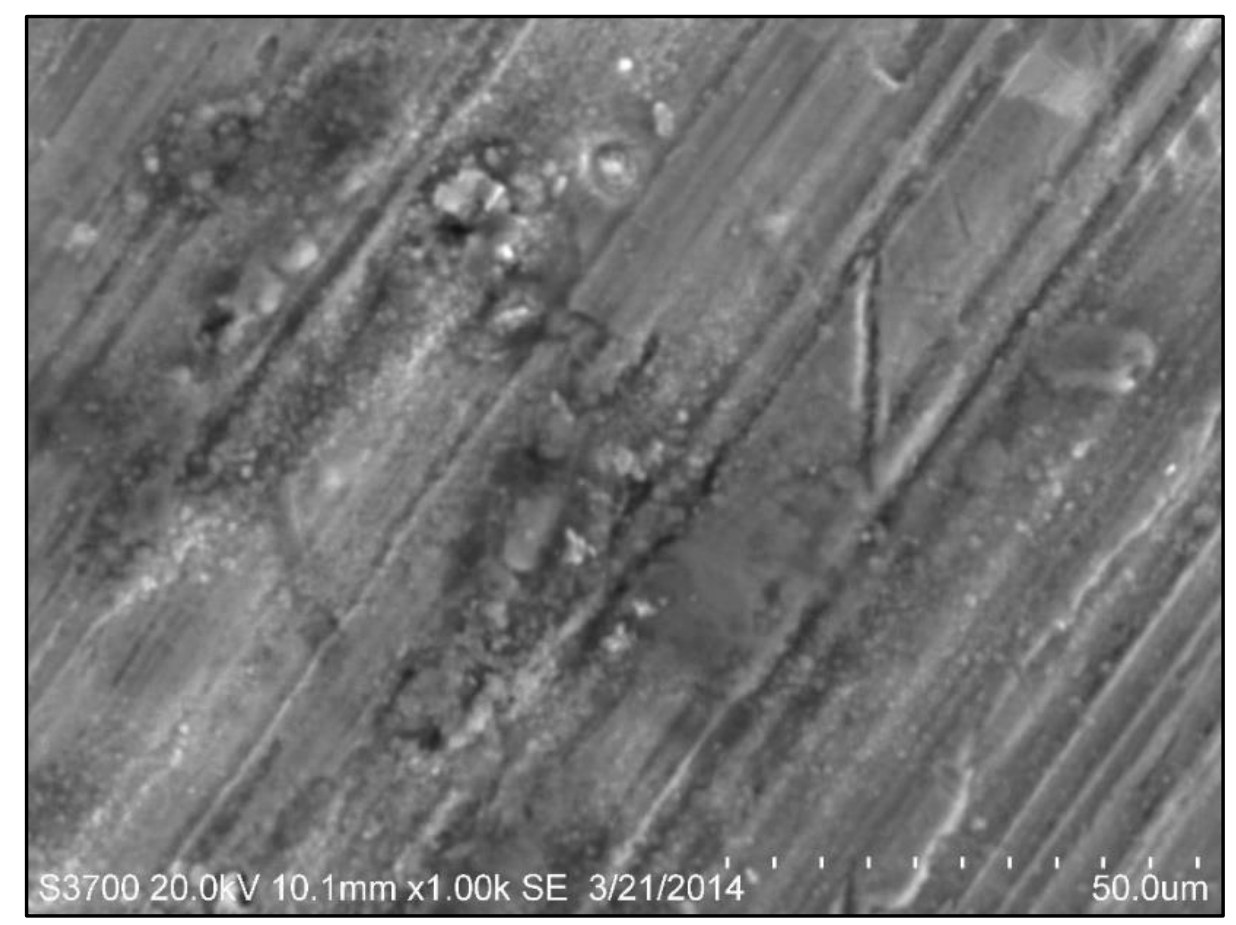

Figure 5: Surface of steel sample post impact testing

Table 10: EDS results from the surface of mild steel sample post liquid impact testing

\begin{tabular}{|l|l|}
\hline Element & Weight\% \\
\hline Nickel, Ni & 10.6 \\
\hline Oxygen, O & 21 \\
\hline Sodium, Na & 7.5 \\
\hline Chlorine, Cl & 4.3 \\
\hline Potassium, K & 1.4 \\
\hline Sulphur, S & 1.6 \\
\hline Manganese, Mn & 0.5 \\
\hline Iron, Fe & 53.1 \\
\hline
\end{tabular}




\section{Discussion}

\subsection{RETR validation}

The rain erosion test rig (RETR) performed as required and a mass loss of mild steel was recorded. The results acquired in this project found the highest mass loss occurred at $16.3 \mathrm{~ms}^{-1}$ of $1.5 \mathrm{mg}$. Developments proved very successful and the hub now rotates with minimum resonance. The hub and whirling arms are more stable being attached using a keyway joint. A new gear has been attached to the rig producing a gear ratio of 1:2 theoretically doubling the velocity in the range of $120 \mathrm{~ms}^{-1}$. Testing was not undertaken using this new gear due to time constraints and difficulties in obtaining a suitable timing belt.

To accommodate the increase in rotational velocity the needles protective shrouds were removed and replaced with longer ones, in an attempt to reduce the effects of the updraft.

Regardless of low mass losses SEM images and EDS results proved rain drop impacts were occurring at the surface of the steel specimens. The main parameter affecting the accuracy of the result was the updraft due to the rotating arms. With the current design of the (RETR) there is no visual aid proving raindrops are colliding with the surface of the test sample. Confirmation of raindrop impacts at high velocities is therefore a vital next step into the research of rain erosion testing using this rig. The most efficient method would be to apply a stroboscope to the rig.

The new mounts demonstrated a quick and simple method of attaching and removing the test samples. The significant downside to the new mount being the effect of galvanic coupling between the test specimen and the mount. Using a non-corrosive coating on the mounts and the bottom surface of the samples proved successful in stopping these reactions between the surfaces. 


\subsection{Effects of linear velocity on the erosion of mild steel}

The results from (Table 4) are presented in (Figure 6) below, indicating a mass loss as the velocity is increased from $16.3 \mathrm{~ms}^{-1}$ to $60.5 \mathrm{~ms}^{-1}$ over 80 minutes of testing. Two test specimens were tested and the mass loss can be seen to increases almost linearly. Both test specimens have a fluctuation in the mass loss at $20 \mathrm{~ms}^{-1}$. The uncertainty in the results from the weighing scales was found to be roughly $\pm 0.1 \mathrm{mg}$ indicated by the error bars in Figure 6 . It was presumed the mass loss vs velocity graph would indicate an increasing gradient as the impact energy increased exponentially with the rising velocity. An increasing impact energy would suggest more impact damage and a larger mass loss. Interestingly McGechaen (2013) discovered a similar trend and from Lee (1990), the mass loss measurements of steel are linearly related to the quantity of ferrite in the microstructure, suggesting a tribo-corrosion mechanism in operation. ${ }^{\text {iv }}$

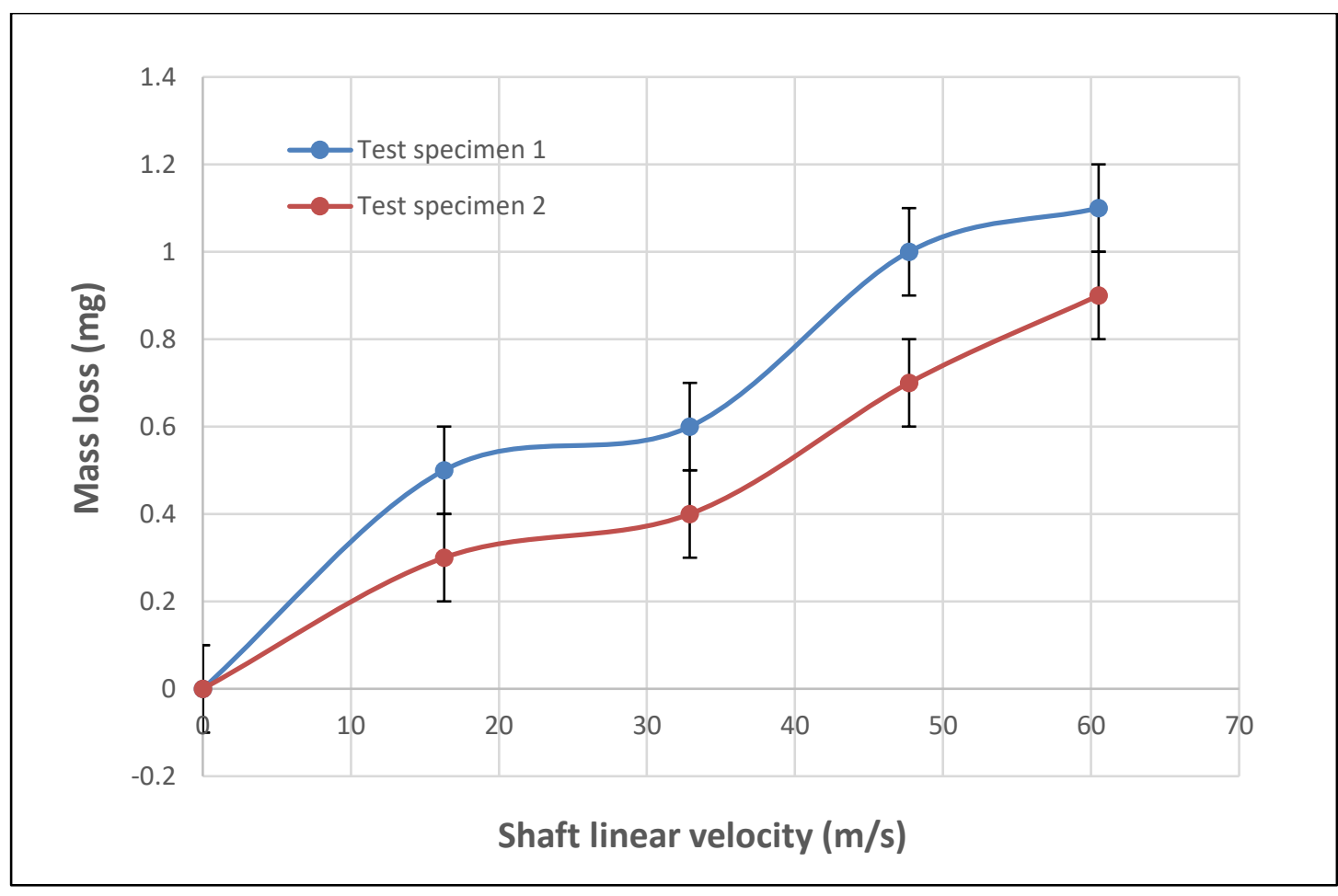

Figure 6: Cumulative mass loss of steel specimens 


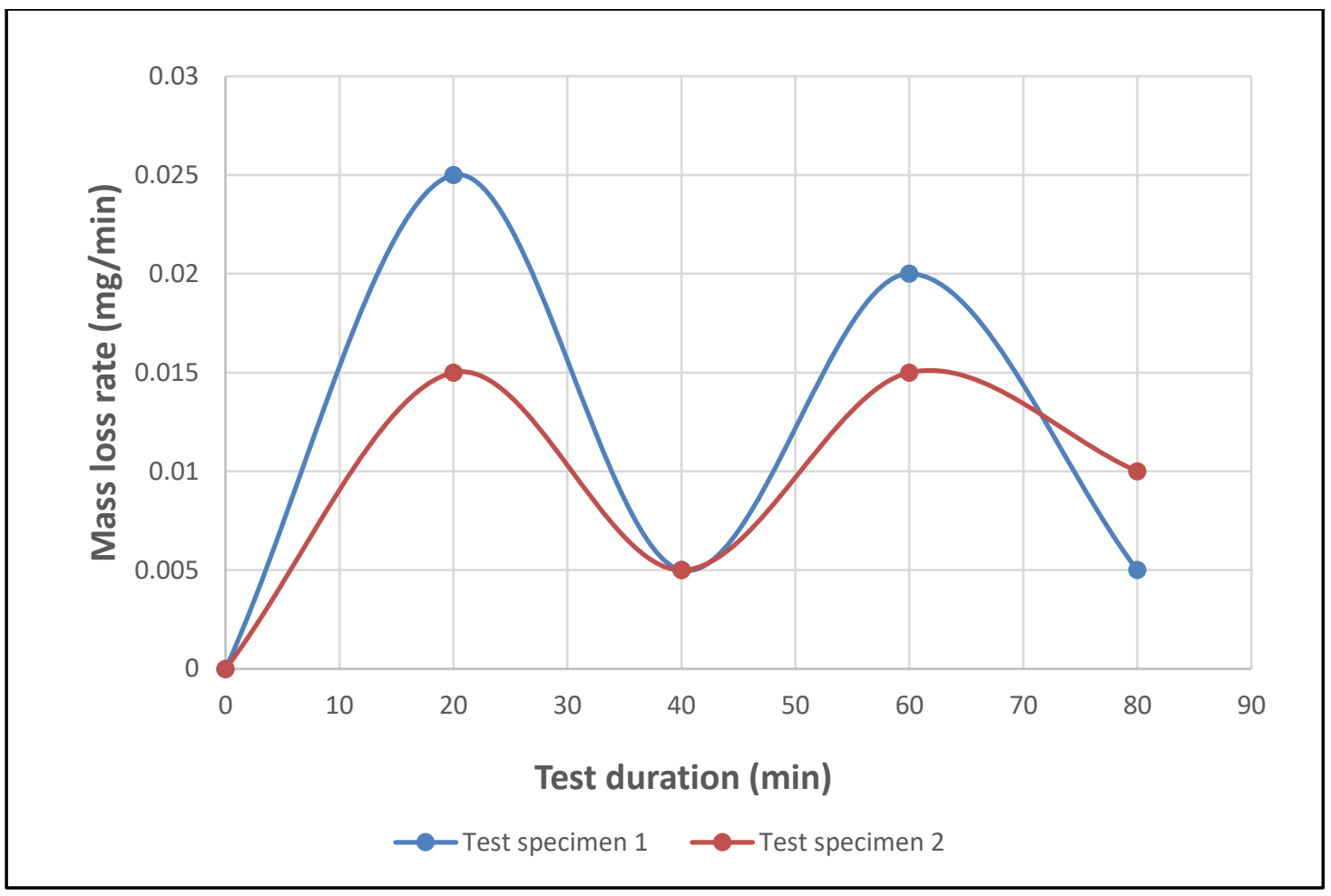

Figure 7: Mass loss rate of steel specimens

In an attempt to explain the effect of increasing linear velocity on the mass loss of mild steel the data was normalized to produce a graph of the mass loss rate during the test, represented in Figure 7. Previous work showed a gradual increase in the mass loss rate with rising linear velocity. ${ }^{14}$ The graph presented in Figure 7 on the contrary indicates a very different trend, between 20 min and 40 $\mathrm{min}$ there is a rapid depletion in mass loss rate. Interestingly during testing in previous work a drop in the mass loss rate was found, although slightly later into the test which could be an indication of an incubation period. ${ }^{\mathrm{xv}}$ Measurements were only taken every 20 minutes, it is quite possible that the incubation period was missed. Lee (1990) had no difficulties and identified an incubation period of roughly 2 minutes for steel containing $0.41 \%$ Carbon. ${ }^{\text {xi }}$ After 40 minutes the mass loss rate starts increasing again signifying erosion of the mild steel as expected after the incubation period, which

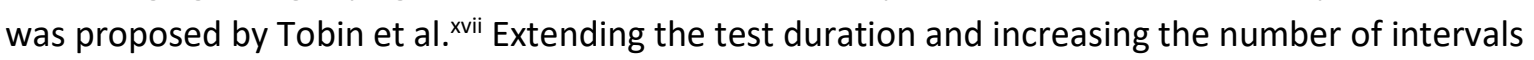
the specimen is weighed would greatly improve the chances of identifying the incubation period.

Unexpectedly (Figure 7) shows another large drop in the mass loss rate 60 minutes into testing. Tobin et al. proposes after the incubation period the wear mechanism tends towards an erosioncorrosion process and the mass loss rate should continue increasing at higher velocities. xviii During testing, visual observations indicated that at high velocities rain droplets would accumulate on the wall of the container and slowly spiral downwards, suggesting many raindrops may not be colliding with the surface of the test specimen. There is no theoretical evidence to verify this theory as the rig is unable to identify raindrop collisions on the surface of the test specimen. A reduction of impacts would certainly contribute to a reduced mass loss rate at higher velocities. 
The following graphs in Figures 8, 9 and 10 represent the total mass at the end of testing for various linear wind velocities, using a constant raindrop diameter. Each test was run for 1 hour and the total mass loss was recorded. All three graphs show signs of the updraft having an effect on the mass loss between $20 \mathrm{~ms}^{-1}-30 \mathrm{~ms}^{-1}$ highlighting a similar conclusion that the updraft must be affecting the number of raindrops colliding with the surface. In all three graphs there is a rise in mass loss at higher wind velocities at around $50 \mathrm{~ms}^{-1}$, this could be related to the increasing impact energy. Although many rain droplets are not colliding with the surface the few that are will be causing greater damage, leading to the increase in mass loss.

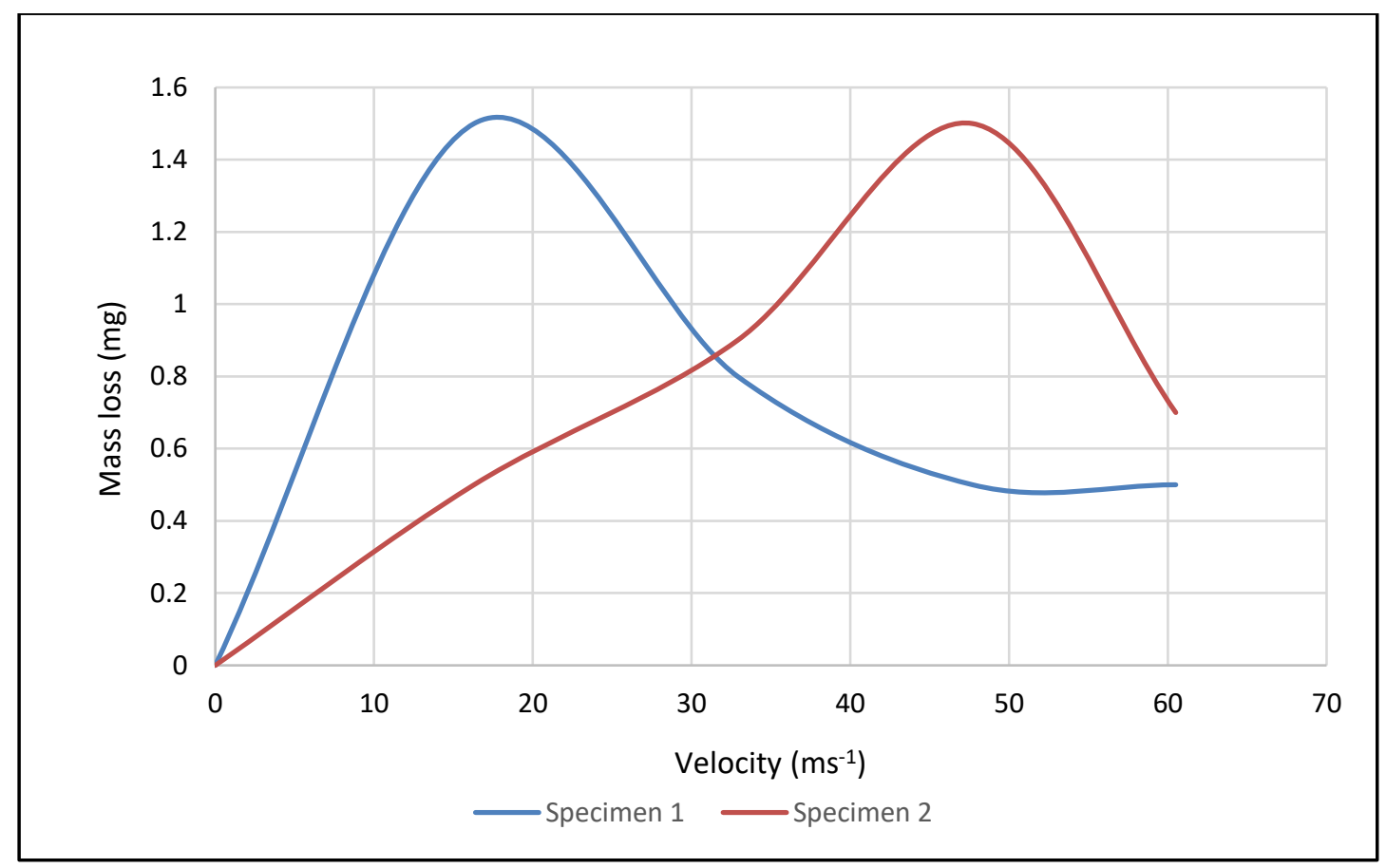

Figure 8: Mass loss at raindrop diameter $2 \mathrm{~mm}$ with increasing linear velocity 


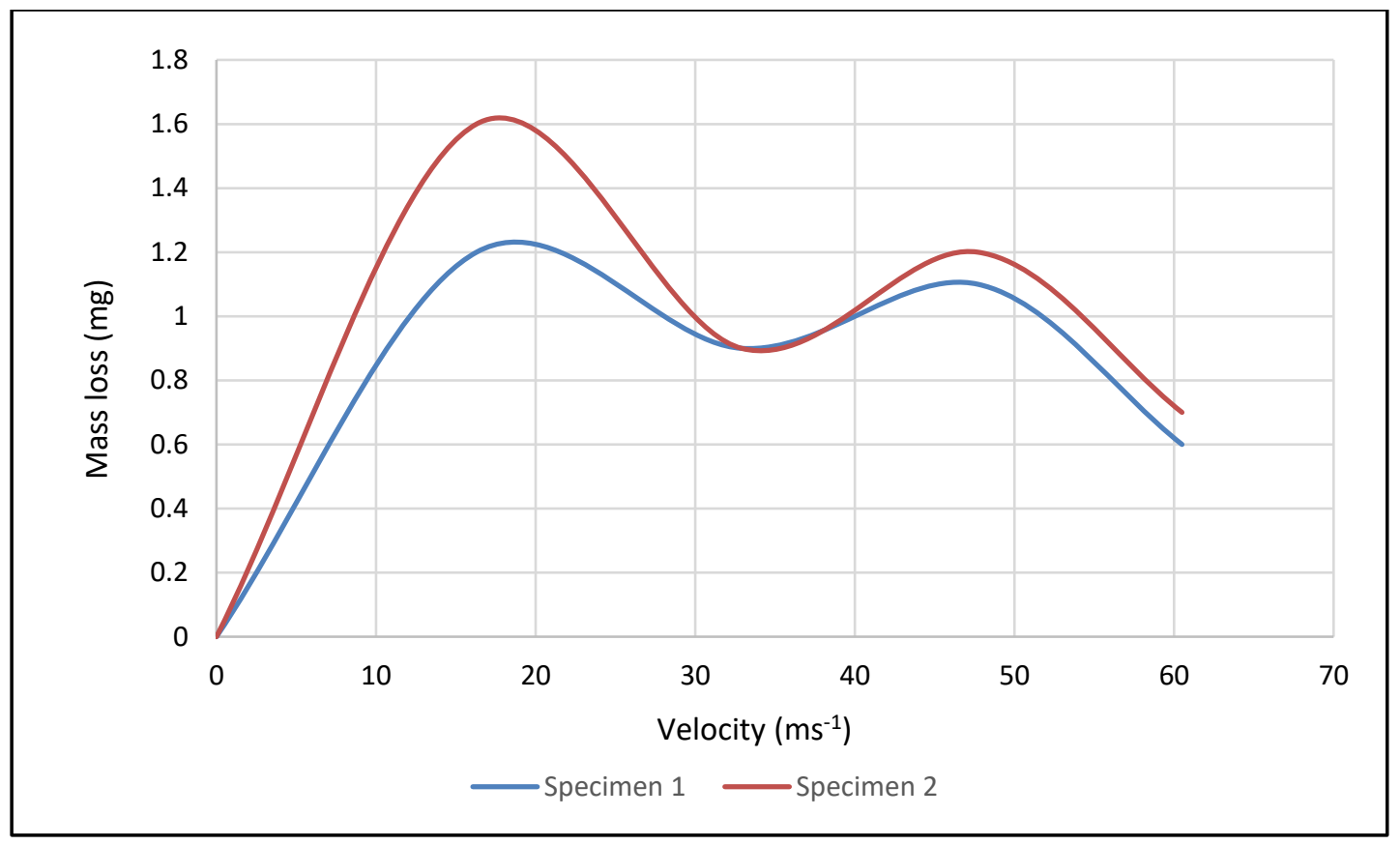

Figure 9: Mass loss at raindrop diameter $2.5 \mathrm{~mm}$ with increasing linear velocity

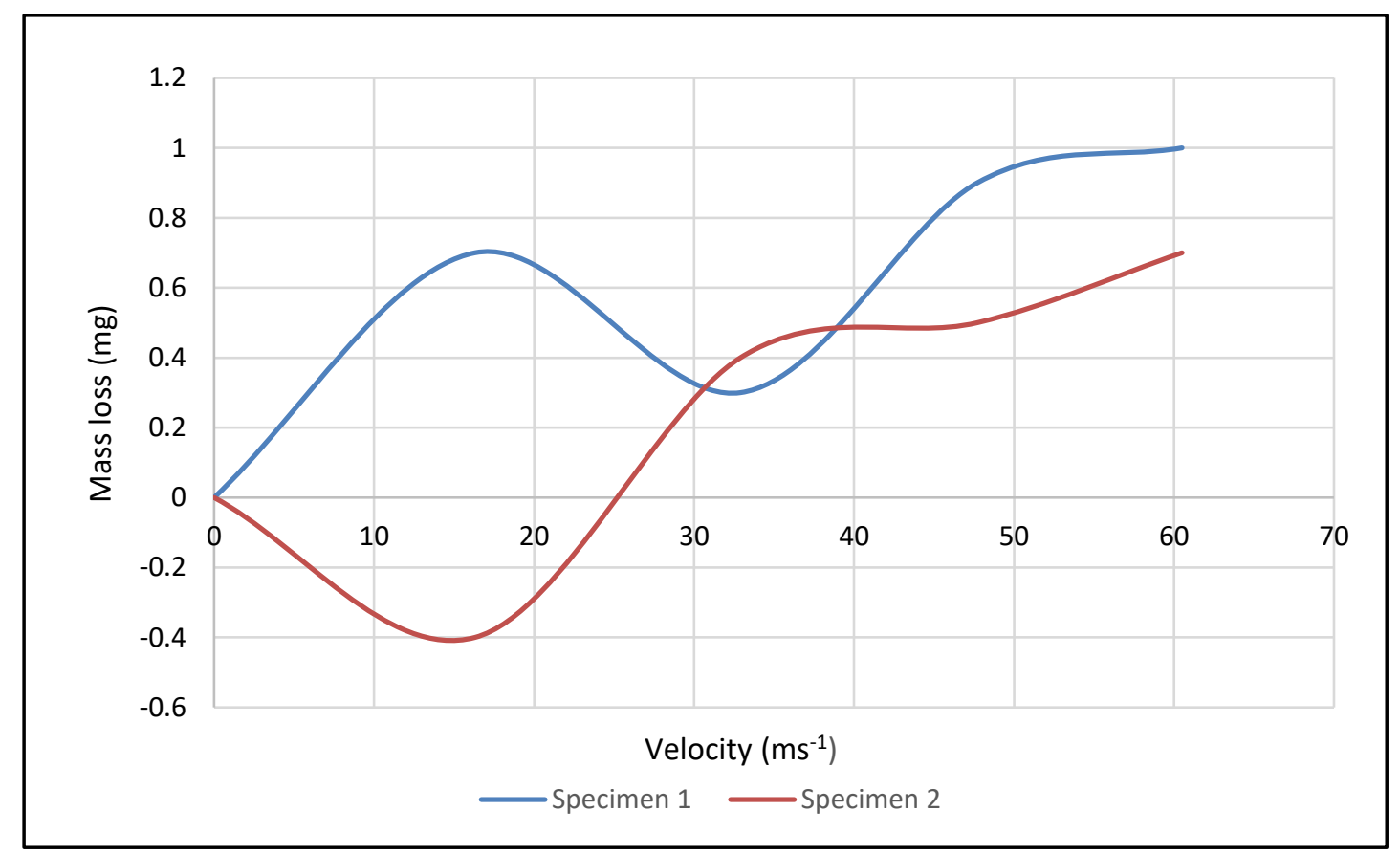

Figure 10: Mass loss at raindrop diameter $2.8 \mathrm{~mm}$ with increasing linear velocity 


\subsection{Effects of raindrop diameter on the erosion of mild steel}

Graphs were created to illustrate the effect of varying raindrop diameters have with increasing linear velocity on the mild steel. Figure 11 shows the effect of varying the raindrop diameter has on the erosion at the surface of mild steel at $16.3 \mathrm{~ms}^{-1}$. The interesting observation made from formulating the data in this format is the mass loss at velocity $16.3 \mathrm{~ms}^{-1}$, clearly shows a decreasing mass loss with increasing rain drop diameter. This was not expected as obviously with increasing diameter the surface area of the rain droplet colliding with the sample is larger, leading to a greater water hammer pressure.

Rain droplet diameters $2.0 \mathrm{~mm}$ and $2.5 \mathrm{~mm}$ can be seen to have a gradual mass loss with evidence of an increasing mass loss rate near 60 minutes. The $2.8 \mathrm{~mm}$ diameter however has an increase in mass at 20 minutes; this could be a sign of the incubation period where an iron oxide layer is forming, acting initially as a protective coating against erosion as has been indicated for elevated temperature

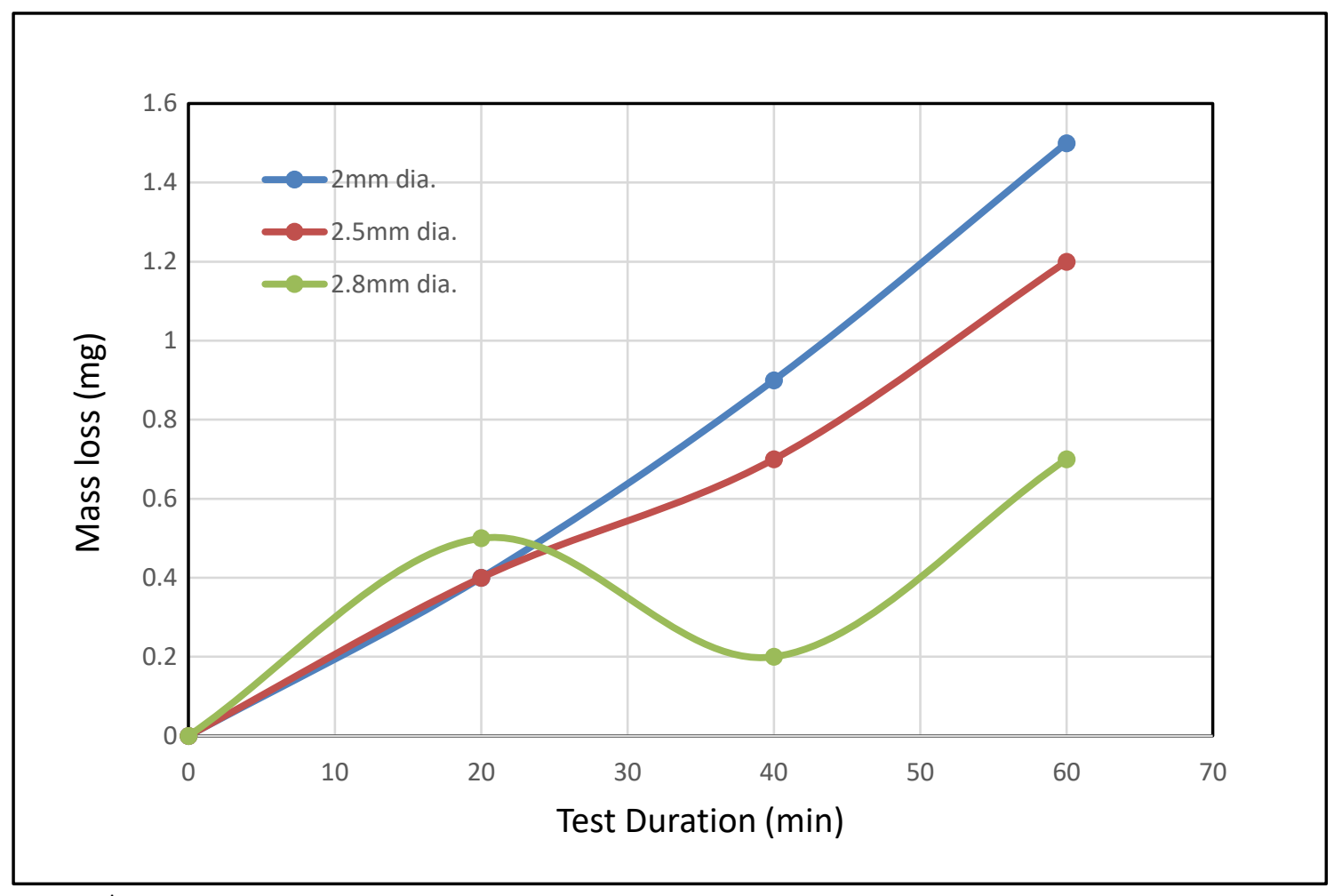

erosion. ${ }^{x i x, x x}$

Referring to (Figure 11) the $2 \mathrm{~mm}$ raindrop diameter provides the greatest damage due to erosion. Possible reasons for this could be that at lower velocities, the updraft is too low to disrupt the trajectory of any raindrop diameter size and with a constant flow rate the smaller gauged needles will produce more rain drops per minute. Perhaps leading to additional impacts on the surface of the specimen, this further bombardment of raindrops will give less time for the surface to cool down 
leading to greater erosion-corrosion. Other work states that the increase in temperature causes a decrease in fluid viscosity resulting in a larger Reynolds number, consequently increasing the erosion. ${ }^{\times x i}$ The temperature on the surface of the material will also have detrimental effect on the material properties of the mild steel such as yield stress, subsequently reducing mechanical resistance. Figure 12 illustrates a very similar trend where the $2.0 \mathrm{~mm}$ diameter raindrops produce the greatest mass loss. However after 40 minutes the mass loss rate of the $2 \mathrm{~mm}$ diameter rain droplets level off, whereas the $2.5 \mathrm{~mm}$ and $2.8 \mathrm{~mm}$ diameters can be seen to increase significantly. Continuing rain drop impact testing after the 40 minute period or incubation period showed rise to greater mass loss rates, as erosion of the iron oxide layer exposes more of the mild steel.

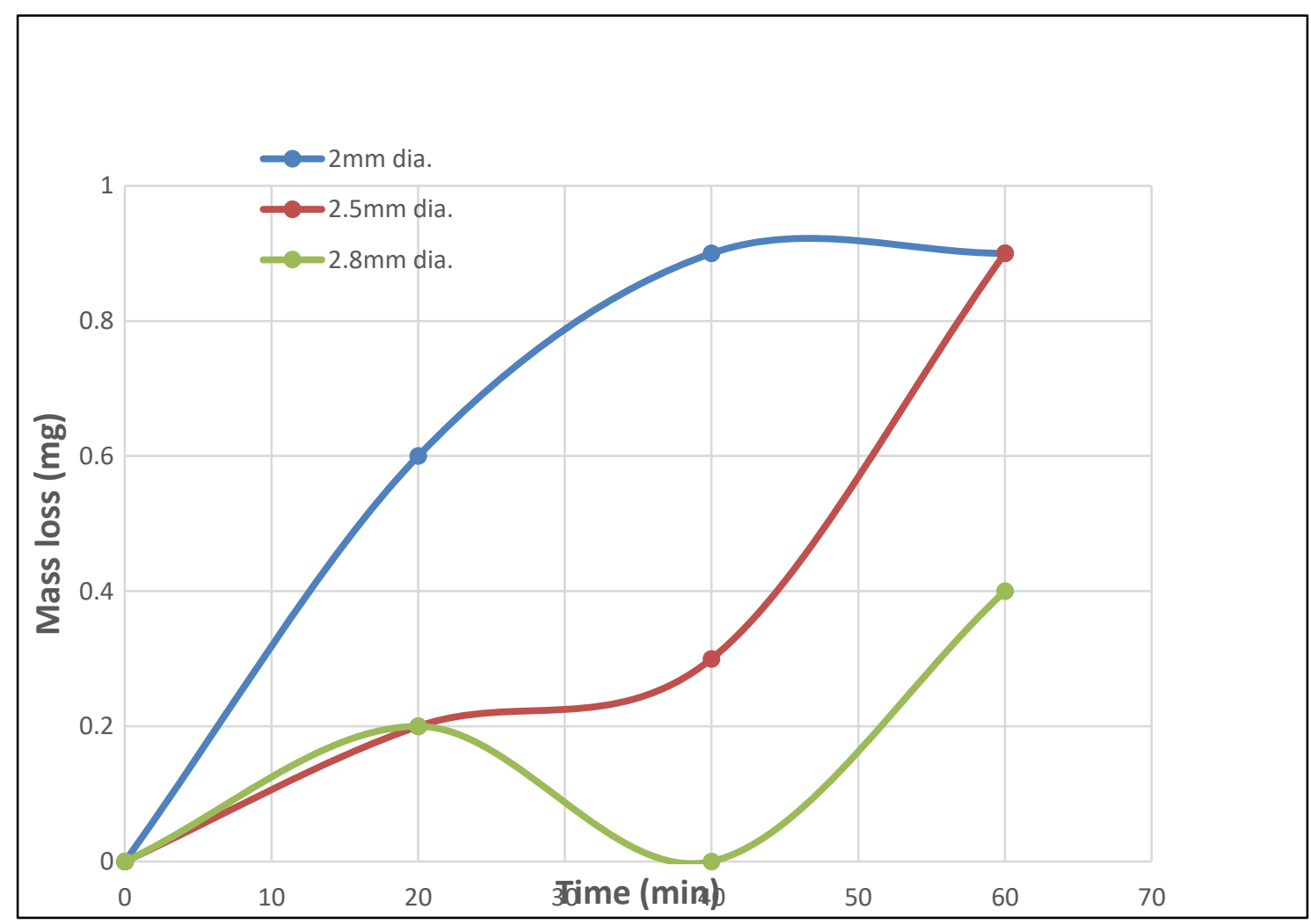

Figure 12: Total mass loss of steel at $32.9 \mathrm{~ms}^{-1}$ with varying rain drop diameter

As the velocity further increases there appears to be a transition phase where the larger raindrop diameters start producing greater mass loss, which is demonstrated in Figures 13 and 14. A theory was that the larger rain droplets are heavier in mass resulting in the updraft having less affect compared to the smaller raindrop diameters. For future research it could be considered to take the weight measurements after 5 minute periods and to increase the test duration, which in theory would improve the accuracy of the graphs and would help determine a more realistic incubation period. 


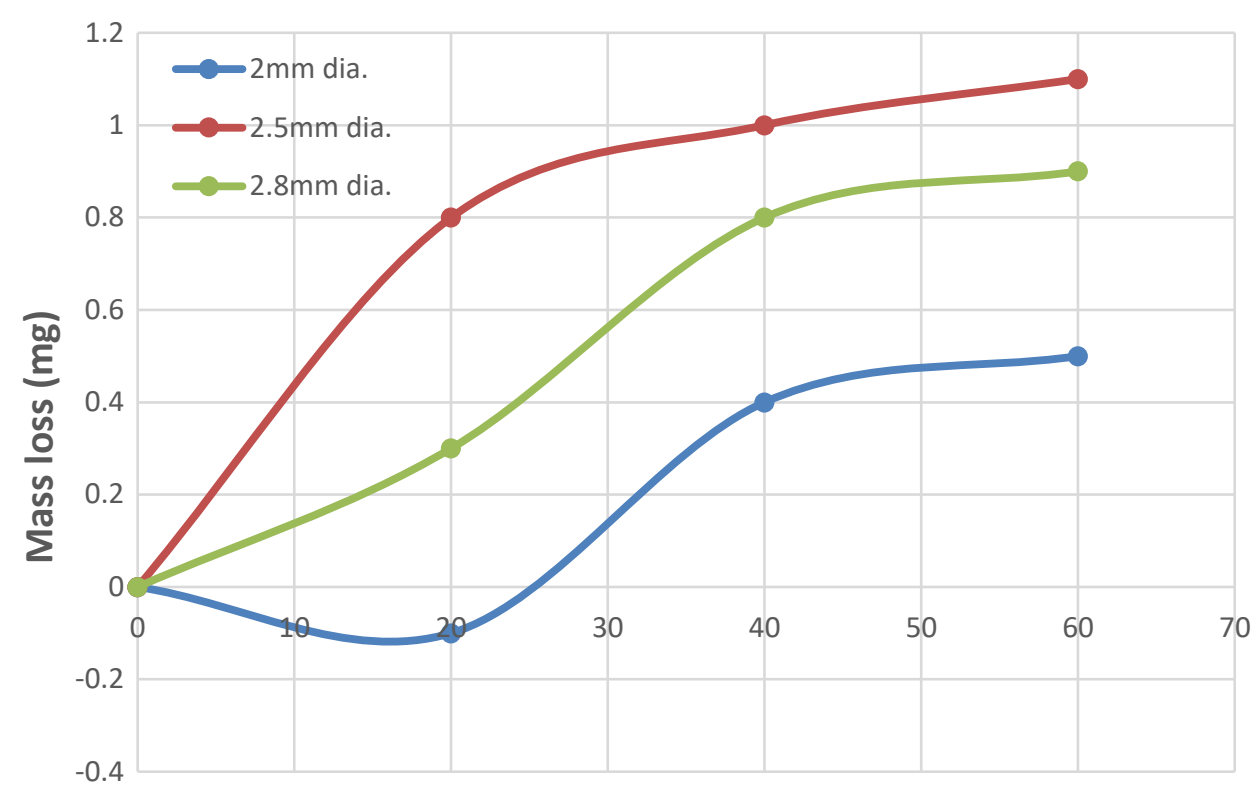

Test Duration (min)

Figure 13: Total mass loss of steel at $47.7 \mathrm{~ms}^{-1}$ with varying rain drop diameter

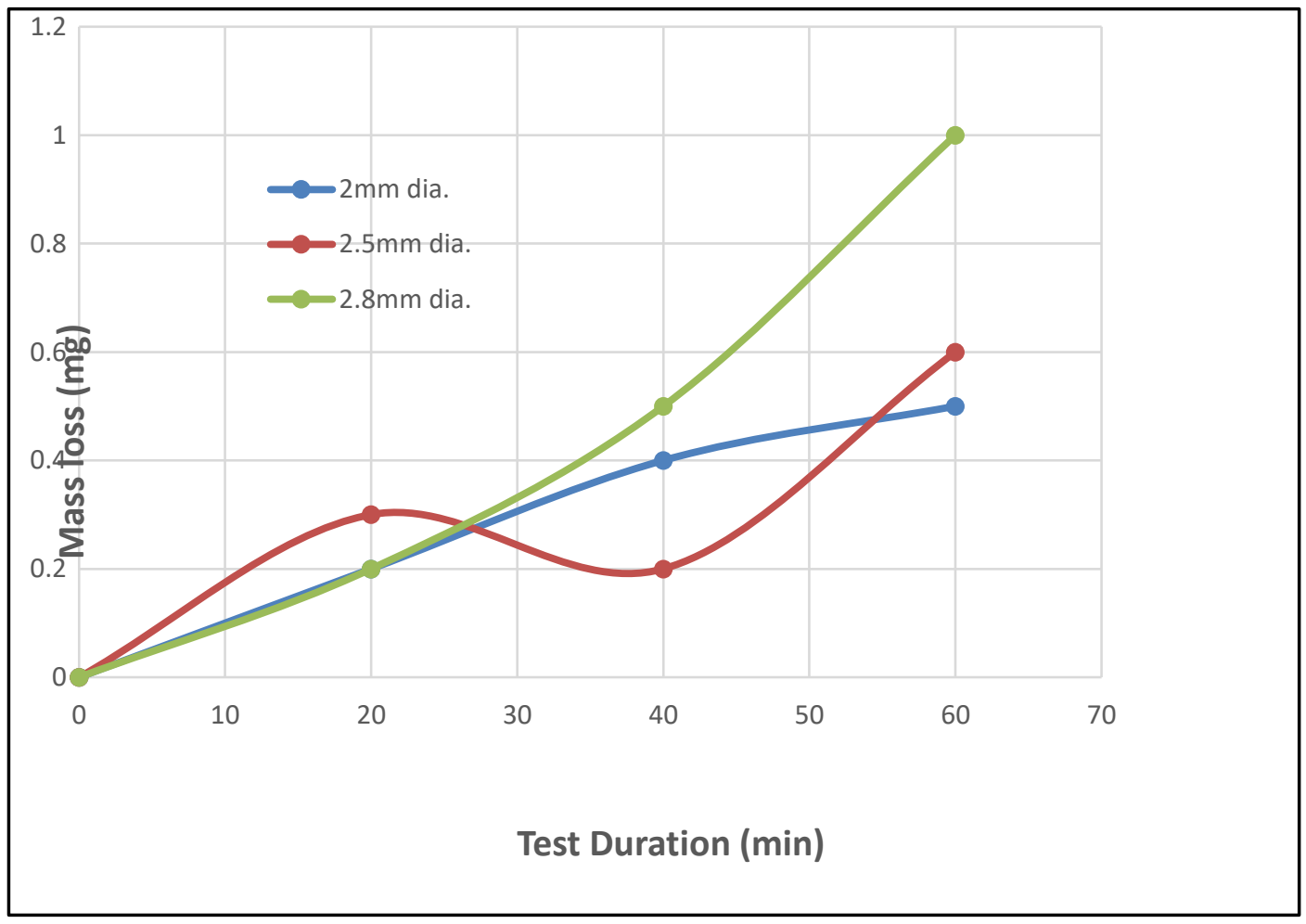

Figure 14: Total mass loss of steel at $60.5 \mathrm{~ms}^{-1}$ with varying rain drop diameter

Figures

$11,12,13$ and 14 confirm an erosion-corrosion process is occurring. M.M. Stack (1991) explains erosion and corrosion are two competing processes that can occur simultaneously on the mild steel 
surface. ${ }^{x x i i}$ During an erosion-corrosion process formation of a passive film acts as a barrier between the mild steel surface and the raindrop impacts causing the oxidation rate of iron to slow while the scale thickens. ${ }^{14,18,21}$

\subsection{SEM and EDS post rain drop impact testing}

From section 4.2, there were clear signs of erosion taking place on the surface of the test specimens due to raindrop impacts. Images were taken with a camera to give a clearer representation of the whole test specimen compared to SEM micrographs, allowing the oxide layer to be seen more clearly.

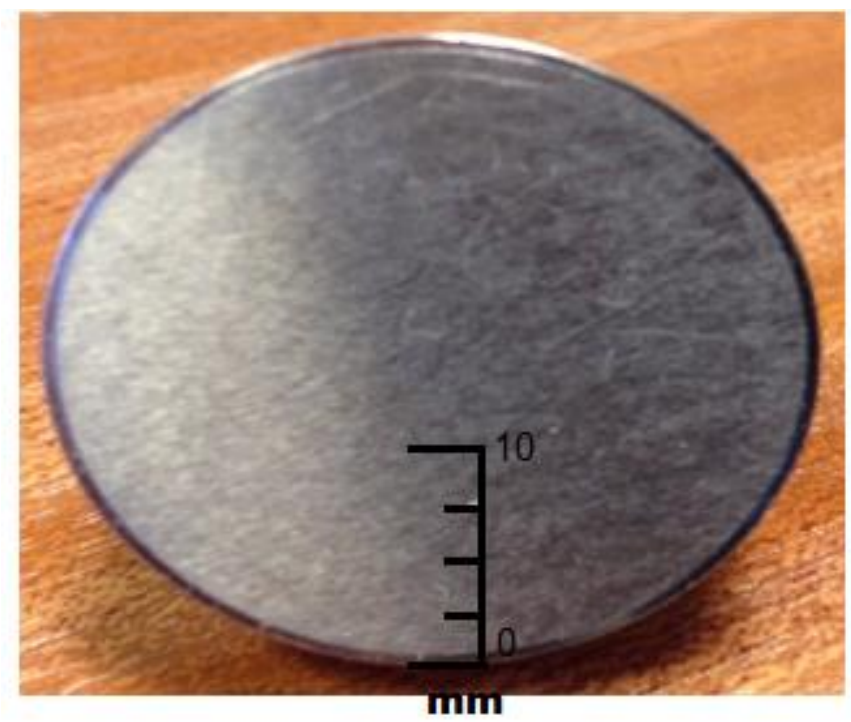

Figure 15: Steel sample prior to impact testing 


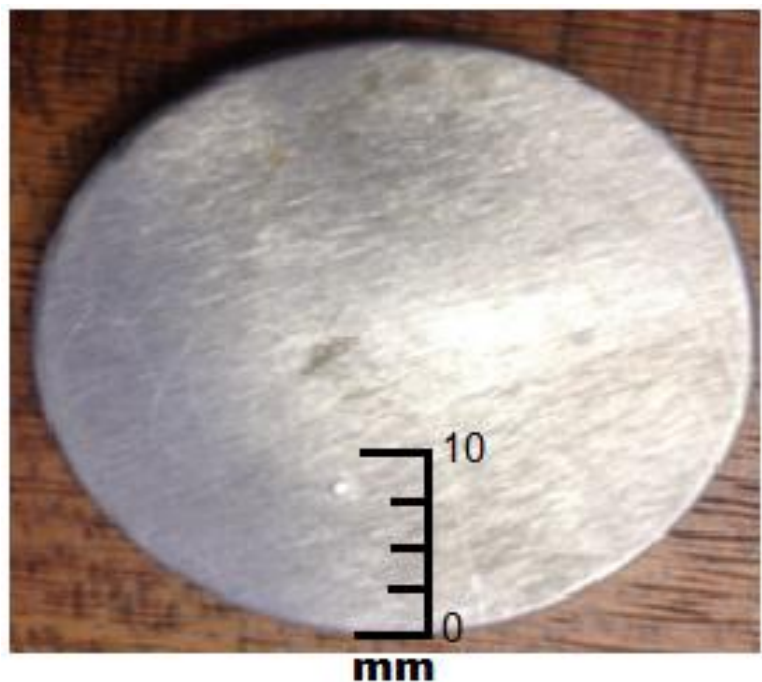

Figure 16: A) Test sample at $16.3 \mathrm{~ms}^{-1}$

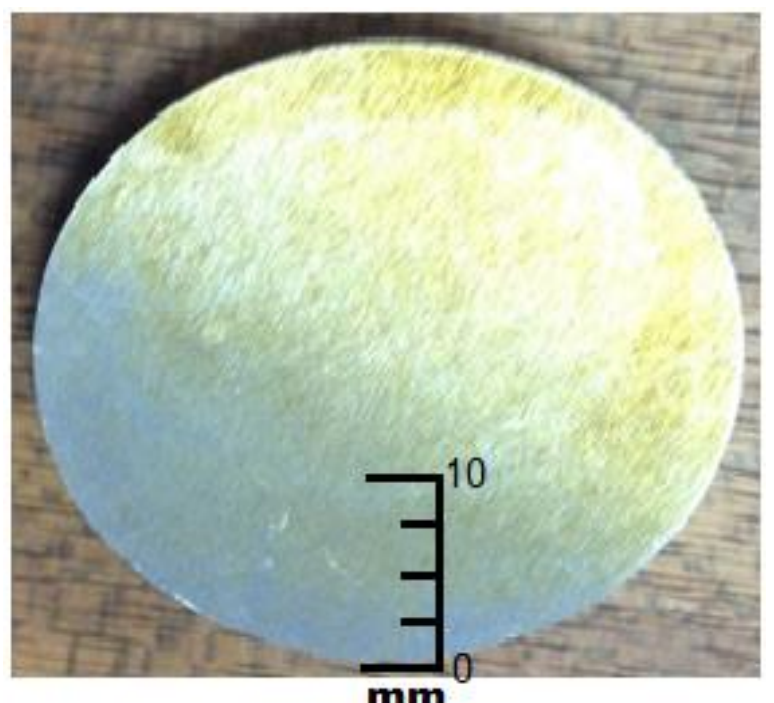

C) Test sample at $47.7 \mathrm{~ms}^{-1}$

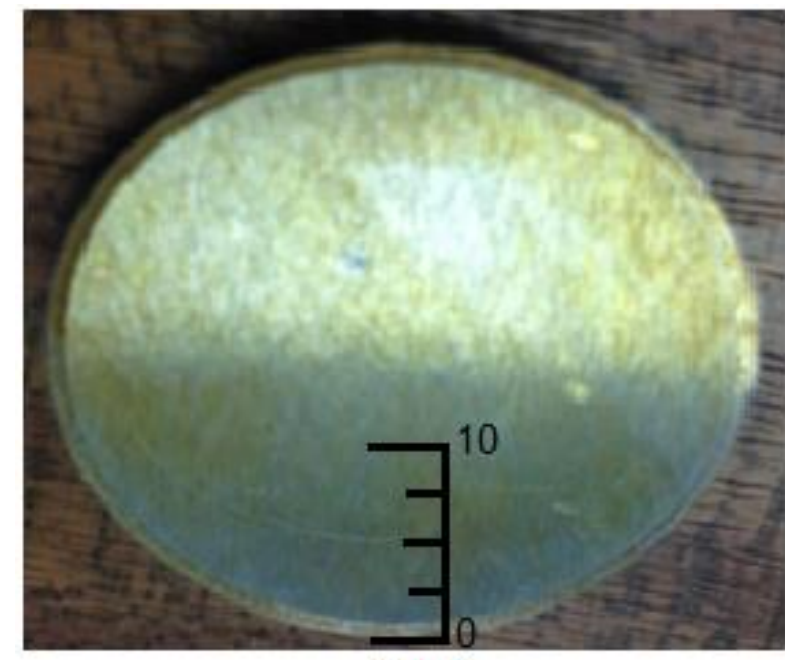

mm

B) Test sample at $32.9 \mathrm{~ms}^{-1}$

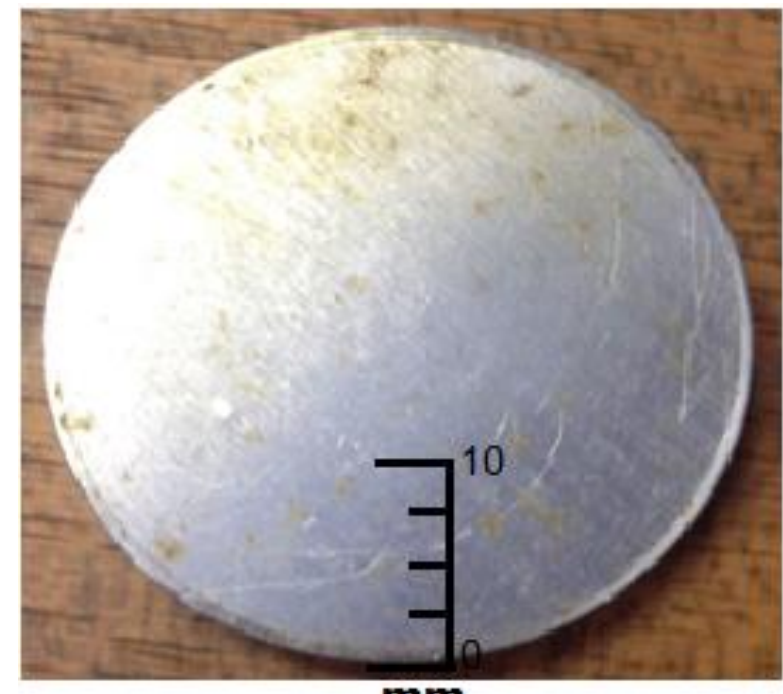

D) Test sample at $60.5 \mathrm{~ms}^{-1}$

Comparing the images after liquid impact testing of various linear velocities at a constant raindrop diameter of $2 \mathrm{~mm}$ (Figure 16), to the image of the samples prior to testing seen in Figure 15, there is evidence on each sample of an oxide layer. The main area affected by the rain drop impacts can be seen at the top half of the sample where there is evidence of the oxide scale initially forming before spreading across the remaining surface. This observation is in agreement with previous work on rain drop liquid impact testing.

Visually from Figure 16[A] there is very little signs of erosion or corrosion occurring on the sample. The mass loss results however show the greatest mass loss coming from this sample under a wind velocity of $16.3 \mathrm{~ms}^{-1}$. Figure $16[\mathrm{~B}]$ shows the iron oxide layer being removed by erosion at $32.9 \mathrm{~ms}^{-1}$ when comparing to Figure $16[\mathrm{C}]$ testing at $47.7 \mathrm{~ms}^{-1}$ the iron oxide layer is much more visible indicating less erosion of the iron oxide scale. Evidence of this can be seen in (Figure 12) where the mass loss rate for testing at $32.9 \mathrm{~ms}^{-1}$ levels off after the 20 minute period however does not yet increase after 60 minutes of testing indicating the oxide layer has not been completely eroded. The 
$47.7 \mathrm{~ms}^{-1}$ test shows an increase in the mass loss rate after 20 minutes of impact testing indicating the end of the incubation period (Figure 13).

SEM micrographs in Figure 17 shows the surface of the oxidised mild steel after liquid impact testing. Several sites of interest have been numbered in the image and (EDS) analysis was run to observe the conditions on the surface at these specific locations. By comparing the results from (Table 9) and (Table 11) it can be seen that a high percentage of oxygen is established on the surface after impact testing however no oxygen was present prior to testing. The heat produced from the raindrop impacts and the wet conditions provided the perfect environment for corrosion to occur. Corrosion was seen visually from images in Figure 16.

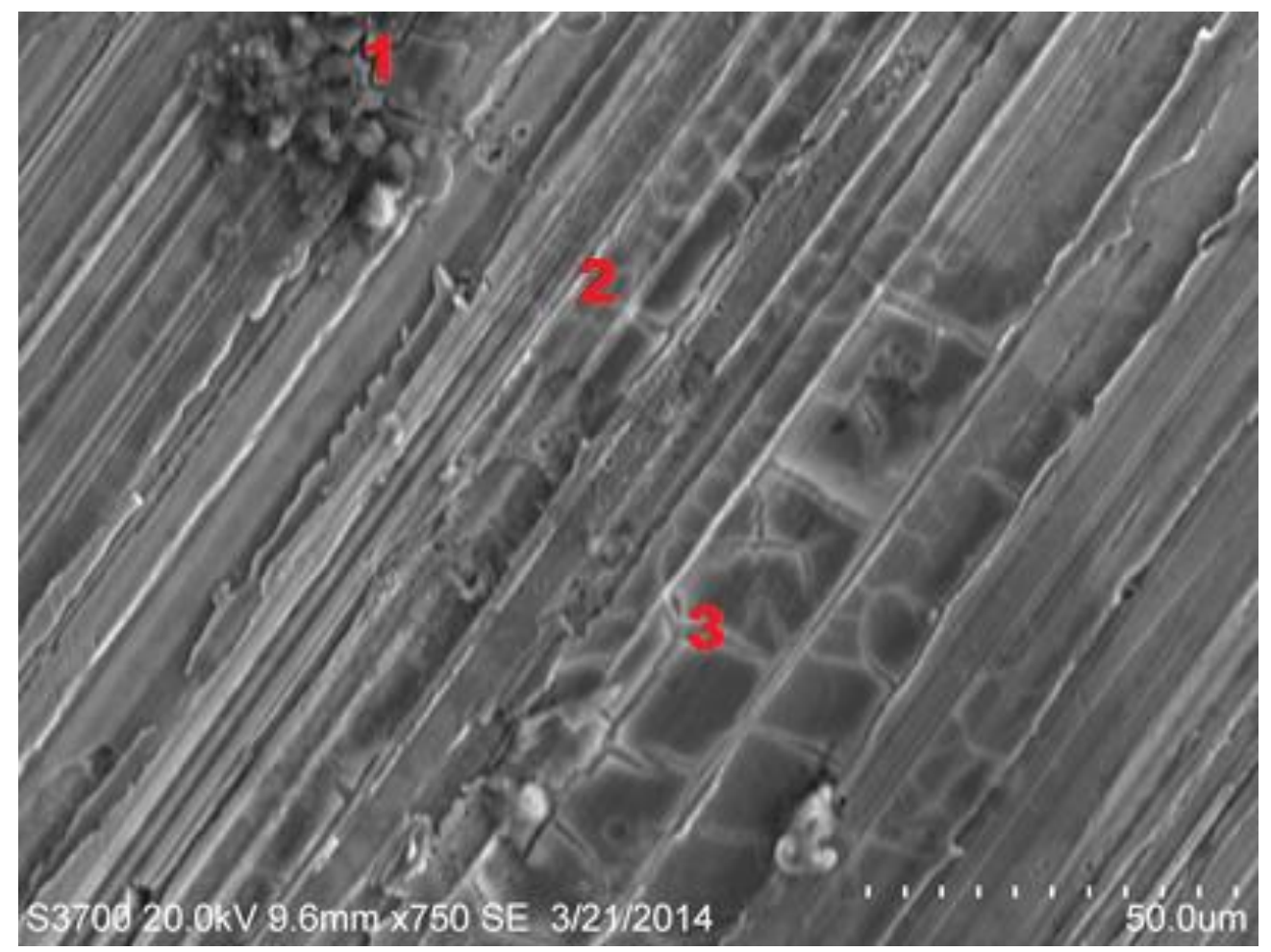

Figure 17: SEM micrograph of oxidised steel

Table 11: Elemental analysis for site 1

\begin{tabular}{|l|l|}
\hline Element & Weight\% \\
\hline Oxygen, O & 26.9 \\
\hline Sodium, Na & 0.89 \\
\hline Chlorine, Cl & 7.21 \\
\hline Potassium, K & 0.56 \\
\hline Sulphur, S & \\
\hline
\end{tabular}




\section{Manganese, Mn}

Iron, Fe
0.54

63.59

The elemental analysis demonstrates the spread of the scale with each site 1, 2 and 3 having variations of oxygen and iron percentages. From Figure 17 site 1 can be seen to be a lot rougher than sites 2 and 3 indicating that corrosion is further developed in this location. Many elements such as potassium, chlorine, copper and sodium are deposited on the surface of the mild steel from the water during testing.

Table 12: Elemental analysis for site 2

\begin{tabular}{|l|l|}
\hline Element & Weight\% \\
\hline Oxygen, O & 5.13 \\
\hline Sodium, Na & 0.74 \\
\hline Copper, Cu & 0.69 \\
\hline Potassium, K & 0.56 \\
\hline Sulphur, S & \\
\hline Manganese, Mn & 0.27 \\
\hline Iron, Fe & 2.41 \\
\hline
\end{tabular}

Table 13: Elemental analysis for site 3

\begin{tabular}{|l|l|}
\hline Element & Weight\% \\
\hline Oxygen, O & 2.46 \\
\hline Sodium, Na & 0.49 \\
\hline Potassium, K & 0.18 \\
\hline Manganese, Mn & 0.59 \\
\hline Iron, Fe & 96.28 \\
\hline
\end{tabular}

Site 3 indicates a low oxygen level of $2.46 \%$ (Table 13) which indicates the scale of the passive film has not developed as much as site 1 which has an oxygen level of $26.9 \%$ (Table 11). From the micrograph formations of stress cracks can be seen at site 3 . This could be an indication of an erosion-corrosion dominant phase where the passive film is being eroded by the liquid impacts. 
Margaret et al. states, erosion begins to enhance corrosion through the removal of an iron oxide layer, as the temperature will increase with repetitive impacts on the surface enhancing the corrosion. ${ }^{22}$ Previous research shows these cracks to be the main mass loss mechanism. ${ }^{14}$

\subsection{Raindrop Erosion maps}

Wastage maps are very important to the tribology field and are used to estimate the magnitude of material loss. These maps show the material loss $(\mathrm{mg})$ considered whether the wastage is low, medium or high. A wastage map was created using the mass losses recorded during testing with respect to varying rain droplet diameter and linear velocity. Previous research on wastage maps for whirling arm rain erosion rigs from the group have addressed this issue, xx111- xxv. Initial parameters for low, medium and high mass loss had to be chosen. Mass loss ranges are as follows;

Low $[<1(\mathrm{mg})]$, Medium $[1-2.5(\mathrm{mg})]$ and High $[>2.5(\mathrm{mg})]$

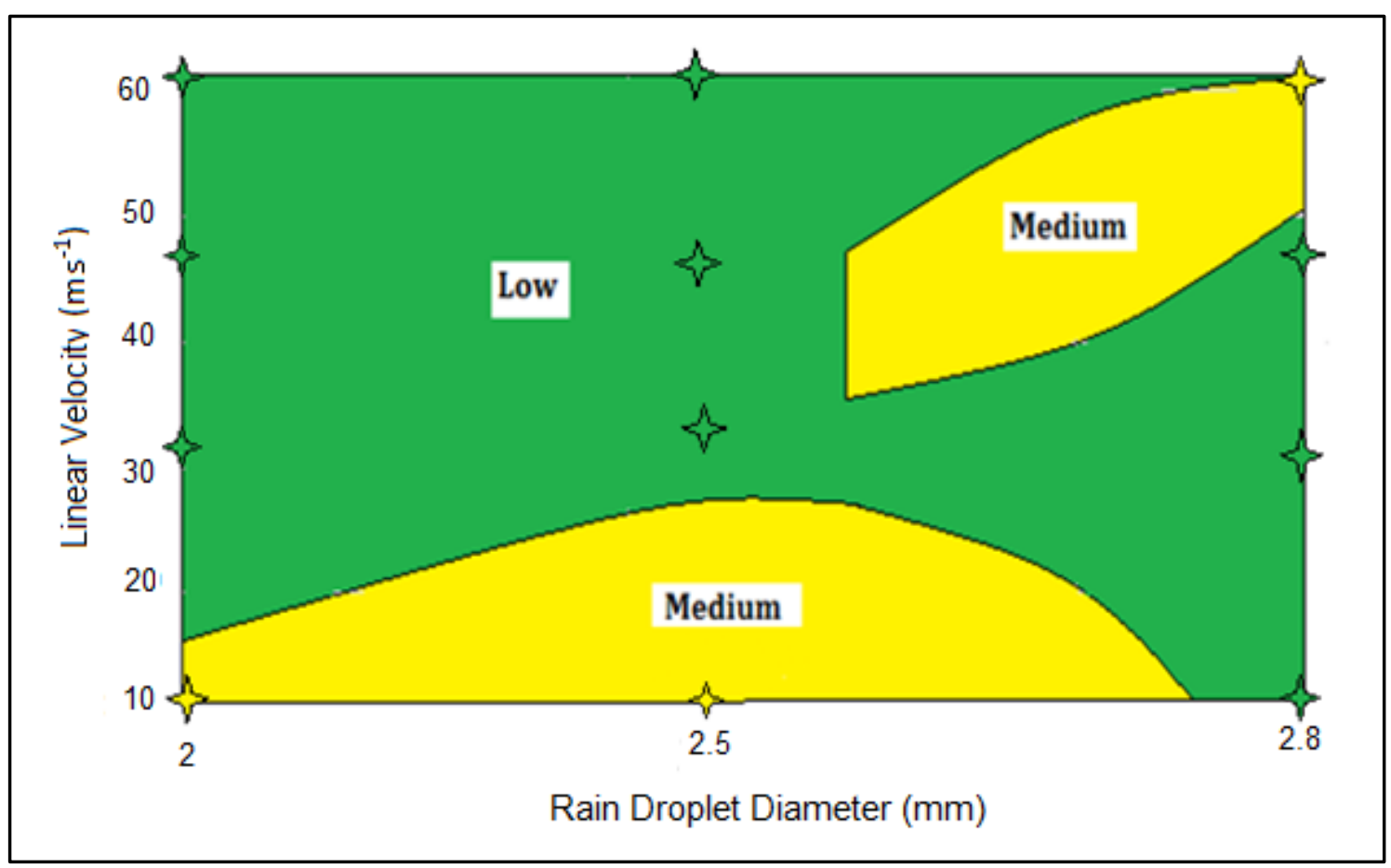

Figure 18: Wastage map for mild steel

Intrestingly the highest mass loss regions were of medium erosion symobolised in (yellow) in Figure 18 above. The largest region of medium mass loss was found at lower velocity between $16.3 \mathrm{~ms}^{-1}$ and $20 \mathrm{~ms}^{-1}$ which was unexpected. The higher mass loss was expected from larger wind velocities as most research from earlier work records peak mass losses at higher velocities. The second medium region can be seen in the top right hand corner of the wastage map. This section is more expected of 
a wastage map for a whirling arm rain erosion test rig as the increasing rain droplet diameter and velocity increases the impact energy on the surface which accelerates erosion. No high mass loss was found during any of the tests on (RETR). The main reason being the linear velocities are very low in comparison to work done by other researchers.

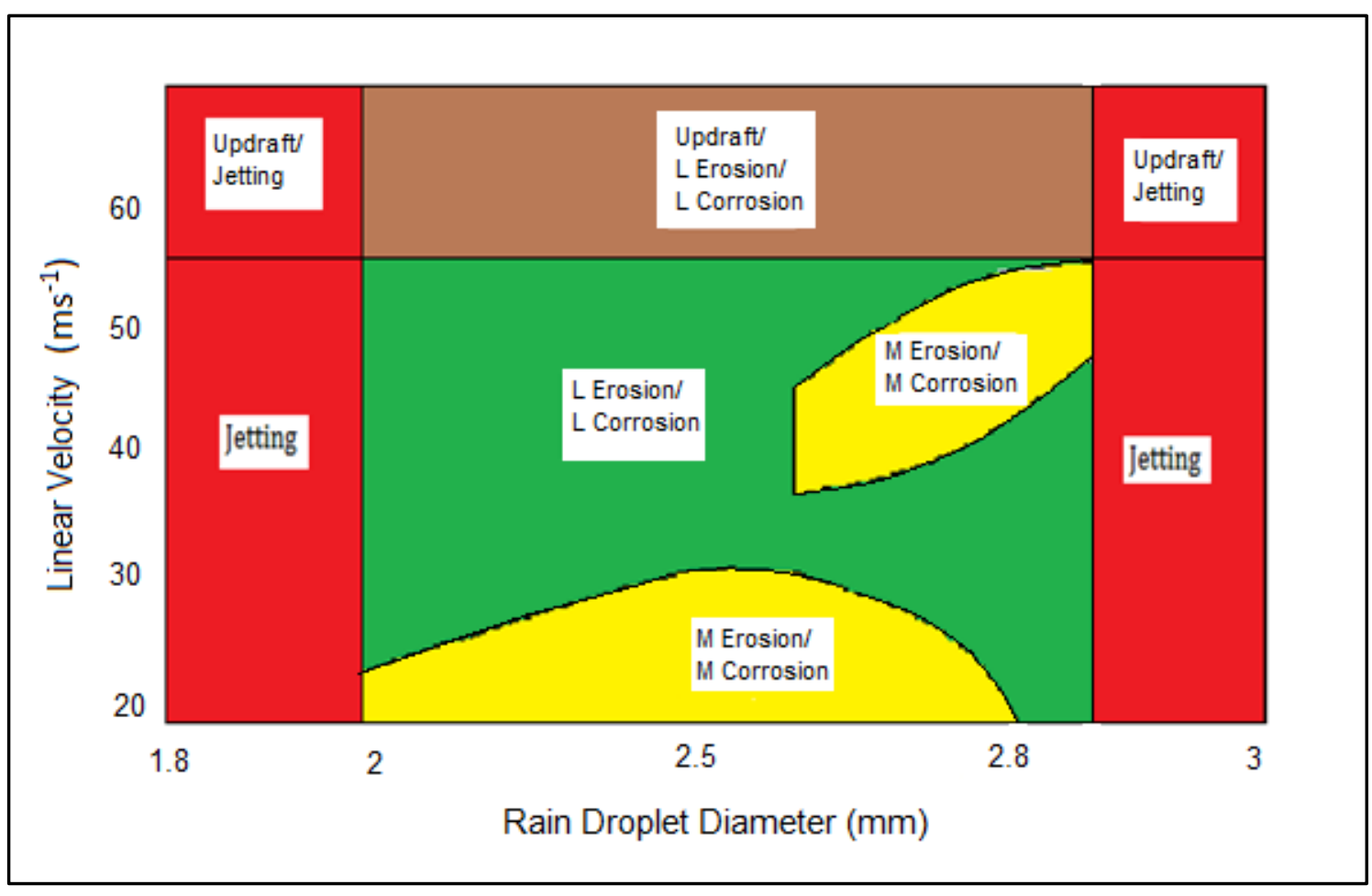

Figure 19: Wastage map showing various raindrop processes at given test conditions

During testing of raindrop diameters $1.8 \mathrm{~mm}$, formation of jets were occurring instead of rain droplets from the ends of the needles. An attempt to produce rain droplets of $1.8 \mathrm{~mm}$ diameter was done by reducing the flow rate to $10 \mathrm{~mL} / \mathrm{min}$, less than half of the required volume flow rate. A reduction in pressure could still not produce rain droplets, so tests had to be voided. A similar problem arose from needles producing $3.0 \mathrm{~mm}$ diameter raindrops, where the formation of water jets would occur from a couple of the needles. Figure 19 is used to illustrate the transition of rain droplet effects encountered during testing caused by jetting from the needles and updraft from the whirling arms. L and M symbolises Low erosion/ corrosion and Medium erosion/ corrosion respectively. 


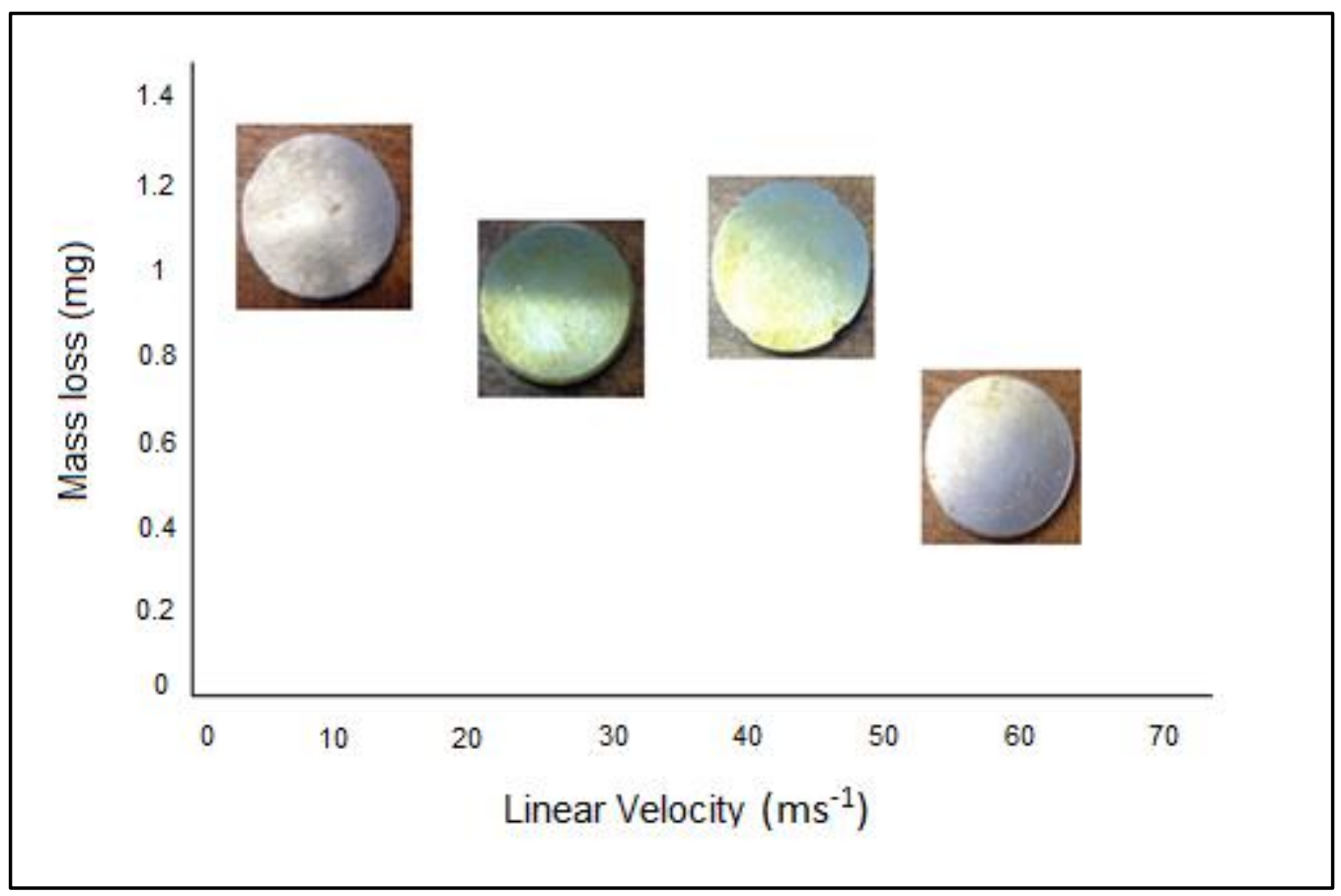

Figure 20: Mass loss graph visualising the effects of corrosion

A further visualization map of the surfaces was produced to compare with the above wastage maps. The graph below (Figure 20) was created to illustrate morphological changes on the surfaces of the specimen. Clearly the surface appearances of corrosion product correspond to areas of higher wastage, Figures. 18 \&19 above.

Hence, the maps indicate that these are potentially useful tools for assessing raindrop erosion mechanisms. The interactions between erosion and corrosion can be identified on the maps and/or the increase in frictional heating of the steel surfaces at the higher velocities. Further work will be to assess in more detail the change in oxide composition over a higher velocity range for this material.

\section{Conclusions}

1) SEM and EDS provide substantial evidence of erosion and the formation of an iron oxide layer signifying an erosion-corrosion process during raindrop erosion of steel.

2) The results indicate that updraft affects the intensity of raindrops colliding with the test specimen surface

3) Erosion wastage maps were created for varying rain droplet diameter and linear wind velocity showing the conditions where raindrop erosion was affected by corrosion and the window of conditions where aerodynamic effects reduced the efficiency of the collosions. 
References

i. $\quad$ Published by TSO (The Stationary Office) (2003): Energy White Paper: Our energy future - creating a low carbon economy. Available at: http://webarchive.nationalarchives.gov.uk

ii. R. Webster, The Carbon Brief Blog (31/10/2013): UK energy statistic show wind power and biomass push renewable electricity to record high. Available at: http://www.carbonbrief.org

iii. Supported by the sixth framework programme (March 2011): Upwind - Design limits and solutions for very large wind turbines. Available at: http://www.upwind.eu/

iv. J.E. Field., (1999) ELSI conference: Invited lecture., Liquid impact: Theory, experiment, applications. WEAR. pp. 233-235 (1-12)

v. M.H. Keegan, D.H. Nash and M.M. Stack., (2013) On erosion issues associated with the leading edge of wind turbine blades. Journal of Physics D: Applied Physics, 46 (38). ISSN 0022-3727

vi. I.M. Hutchings., (1992) Ductile-brittle transitions and wear maps for the erosion and abrasion of brittle materials. Jounal of Physics D: Applied Physics. 25 (1A) pp. A212-A221

vii. S. Hattori., M. Takinami., (2010) Comparison of cavitation erosion rate with liquid impingement erosion rate. WEAR. 269 pp. 310-316.

viii. K. Wood (2011) Blade Repair: Closing the maintenance gap. Composites technology. Available at: http://www.compositesworld.com

ix. $\quad 3 \mathrm{M}$ Wind Energy: History and Innovations of 3M. Available at:

http://solutions.3m.com

x. A. Kubilay, a, b., D. Derome, a, b., B. Blocken, c., J. Carmeliet, a, b., (2013) CFD simulation and validation of wind-driven rain on a building façade with an Eulerian multiphase model. Building and Environment. (61) pp. 69-81

xi. A.C. Best (1950) The size distribution of raindrops. Q J R Meteorological Soc, (76) pp. $16-36$ 
xii. A.C. Best (1950) The size distribution of raindrops. Q J R Meteorological Soc, (76) pp. $16-36$

xiii. M.M. Stack, and G.H. Abdulrahmen, (2010) Mapping erosion-corrosion of carbon steel in oil exploration conditions : some new approaches to characterizing mechanisms and synergies. Tribology International, 43 (7). pp. 1268-1277.

xiv. R. McGechaen. (2013) MEng (University of Strathclyde

xv. N. Krishnamurthy, M.S. Murali, B.Venkataraman and P.G. Mukunda (2012) Erosion behaviour of plasma sprayed alumina and Calcia-Stabilized Zirconia coatings on cast iron substrate, ceramic coatings - Applications in Engineering, Prof. Freg Shi (ED.), ISBN: 978953-0083-6, InTech,.

Available at: http://www.intechopen.com/books/ceramic-coatings-applications-inengineering

xvi. G.M.C. Lee (1990) The erosion resistance of plain steels under water droplet impacts conditions. Wear. 141 pp. 185-201

xvii. E.F. Tobin, T.M. Young, D. Raps, O. Rohr,. (2011) Comparison of liquid impingement results from whirling arm and water-jet rain erosion test facilities - WEAR, 271. pp. 2625 2631

xviii. E.F. Tobin, T.M. Young, D. Raps,. (2012) Evaluation and correlation laboratory results from a rain erosion test campaign. $28^{\text {th }}$ International congress of the aeronautical sciences.

Available at: http://www.icas.org

xix. I. Finnie (1972) Some observations on the erosion of ductile materials: Wear, 19 (1972) 81-90.

xx. M.M. Stack, F.H. Stott and G.C. Wood (1993) Review of mechanisms of erosioncorrosion of alloys at elevated temperatures. WEAR, 162-164 (B). pp. 706-712.

xxi. M.M. Stack, M.S. Abdelrahman and B. Jana,. (2010) Some perspectives on modelling the effect of temperature on the erosion-corrosion of FE in aqueous conditions. Tribology International, 43 (12). pp. 2279-2297. ISSN 0301679X 
xxii. M.M. Stack, F. H. Stott and G. C. Wood,. (1992) The effect of pre-oxidation of chromia and alumina forming alloys on erosion in laboratory simulated fluidized-bed conditions. Corrosion Science, Vol. 33. (6), pp. $965-983$

xxiii G Rasool, A.C. Middleton, and M.M.Stack, (2020) Mapping raindrop erosion of GFRP composite wind turbine blade materials : perspectives on degradation effects in offshore and acidic environmental conditions. Journal of Tribology, 142 (6). TRIB-19-1279. ISSN 07424787

xxiv K Pugh, Kieran, G Rasool and M.M. Stack (2019) Raindrop erosion of composite materials : some views on the effect of bending stress on erosion mechanisms. Journal of Bio- and Tribo-Corrosion, 5 (2). 45. ISSN 2198-4220

xxv K Pugh, G Rasool, and M.M. Stack (2018) Some thoughts on mapping tribological issues of wind turbine blades due to effects of onshore and offshore raindrop erosion. Journal of Bio- and Tribo-Corrosion. pp. 1-8. ISSN 2198-4220. 